\title{
A NEW MULTISCALE FINITE ELEMENT METHOD FOR HIGH-CONTRAST ELLIPTIC INTERFACE PROBLEMS
}

\author{
C.-C. CHU, I. G. GRAHAM, AND T.-Y. HOU
}

\begin{abstract}
We introduce a new multiscale finite element method which is able to accurately capture solutions of elliptic interface problems with high contrast coefficients by using only coarse quasiuniform meshes, and without resolving the interfaces. A typical application would be the modelling of flow in a porous medium containing a number of inclusions of low (or high) permeability embedded in a matrix of high (respectively low) permeability. Our method is $H^{1}$ - conforming, with degrees of freedom at the nodes of a triangular mesh and requiring the solution of subgrid problems for the basis functions on elements which straddle the coefficient interface but which use standard linear approximation otherwise. A key point is the introduction of novel coefficientdependent boundary conditions for the subgrid problems. Under moderate assumptions, we prove that our methods have (optimal) convergence rate of $O(h)$ in the energy norm and $O\left(h^{2}\right)$ in the $L_{2}$ norm where $h$ is the (coarse) mesh diameter and the hidden constants in these estimates are independent of the "contrast" (i.e. ratio of largest to smallest value) of the PDE coefficient. For standard elements the best estimate in the energy norm would be $\mathcal{O}\left(h^{1 / 2-\varepsilon}\right)$ with a hidden constant which in general depends on the contrast. The new interior boundary conditions depend not only on the contrast of the coefficients, but also on the angles of intersection of the interface with the element edges.
\end{abstract}

\section{INTRODUCTION}

In this paper we present a new application of multiscale finite element methods for the classical elliptic problem in weak form

$$
\int_{\Omega} \mathcal{A}(x) \nabla u(x) \cdot \nabla v(x) d x=\int_{\Omega} F(x) v(x) d x, \quad v \in H_{0}^{1}(\Omega),
$$

where the solution $u \in H^{1}(\Omega)$ is required to satisfy a Dirichlet condition on $\partial \Omega$ and $F$ is given on a bounded domain $\Omega \subset \mathbb{R}^{2}$. To concentrate on the essential aspects of this new theory we primarily treat the homogeneous Dirichlet problem when the boundary of $\Omega$ is a convex polygon. (These are not essential restrictions: All our results are true for smooth boundaries as well. Similar results could be obtained for

Received by the editor February 24, 2009.

2010 Mathematics Subject Classification. Primary 65N12, 65N30.

Key words and phrases. Second-order elliptic problems, interfaces, high contrast, multiscale finite elements, non-periodic media, convergence.

The authors thank Rob Scheichl and Jens Markus Melenk for useful discussions. The second author acknowledges financial support from the Applied and Computational Mathematics Group at California Institute of Technology. The research of the third author was supported in part by an NSF Grant DMS-0713670 and a DOE Grant DE-FG02-06ER25727. 
non-convex polygons, treated with local mesh refinement. Moreover, we state the corresponding results for inhomogeneous boundary conditions later in the paper.)

The coefficient $\mathcal{A}$, which is assumed here to be scalar, will be allowed to jump across a number of smooth interior interfaces, and the aim of the present paper is to propose and analyse a new multiscale finite element method for this problem on (coarse) meshes which are not required to resolve the interfaces. Our method has the same rate of convergence (with respect to mesh diameter) as is known for the case when $\mathcal{A}$ is globally smooth (i.e. the rate is not degraded by the loss of solution regularity across the interfaces) and, moreover (in a way made precise below), this rate of convergence is independent of the range of variation ("contrast") of the coefficient function $\mathcal{A}$.

While the method we propose could be used for the case when the interfaces in $\mathcal{A}$ are of general geometry, our proofs will be given for the particular case when $\Omega$ contains a finite number of inclusions, each with smooth closed boundary not intersecting $\partial \Omega$, such as is depicted in Figure 1, Denoting the inclusions by $\Omega_{1}, \ldots, \Omega_{m}$ and setting $\Omega_{0}=\Omega \backslash \bigcup_{i=1}^{m} \Omega_{i}$, we assume that the coefficient $\mathcal{A}$ is piecewise constant with respect to the decomposition $\left\{\Omega_{i}: i=0, \ldots, m\right\}$. (Again there is no essential difficulty in generalising to piecewise smooth coefficients.) Setting $\mathcal{A}_{\text {min }}=\min \left\{\left.\mathcal{A}\right|_{\Omega_{i}}: i=0, \ldots, m\right\}$, we first scale problem (1.1) by dividing by $\mathcal{A}_{\text {min }}$, yielding the weak form: Find $u$ such that $u \in H_{0}^{1}(\Omega)$, and

$$
a(u, v)=(f, v)_{L_{2}(\Omega)} \text { for all } v \in H_{0}^{1}(\Omega),
$$

where

$$
a(u, v)=\int_{\Omega} \alpha(x) \nabla u(x) \cdot \nabla v(x) d x
$$

with

$$
\alpha(x)=\frac{1}{\mathcal{A}_{\min }} \mathcal{A}(x), \quad f(x)=\frac{1}{\mathcal{A}_{\min }} F(x) .
$$

Clearly then, $\alpha$ is piecewise constant with respect to the partition $\left\{\Omega_{i}: i=\right.$ $0, \ldots, m\}$ and $\alpha(x) \geq 1$ for all $x \in \Omega$. Letting $\alpha_{i}$ denote the restriction of $\alpha$ to $\Omega_{i}$, our analysis will focus on the proof of robust optimal order convergence in each of the two "high contrast" cases, characterised by a large "contrast parameter" $\widehat{\alpha}$ :

$$
\begin{array}{cc}
\text { Case I : } & \widehat{\alpha}:=\min _{i=1, \ldots, m} \alpha_{i} \rightarrow \infty, \quad \alpha_{0}=1, \\
\text { Case II : } & \widehat{\alpha}:=\alpha_{0} \rightarrow \infty, \max _{i=1, \ldots, m} \alpha_{i} \leq K,
\end{array}
$$

for some constant $K$. In Case I, the inclusions are high permeability compared to the background matrix, while Case II contains the converse configuration.

Our method will involve special "multiscale" nodal basis functions on a (coarse) quasiuniform triangular mesh $\mathcal{T}_{h}$. On elements on which $\alpha$ is constant, these basis functions just coincide with the usual linear hat functions. Otherwise we precompute the basis function by solving (approximately) a homogeneous version of (1.2) on the relevant elements, subject to special boundary conditions described later in this paper. The resulting basis functions are then used to define a multiscale finite element solution $u_{h}^{\mathrm{MS}}$ by the usual Galerkin method. We show that our method satisfies an error estimate of the form

$$
\left|u-u_{h}^{\mathrm{MS}}\right|_{H^{1}(\Omega)} \leq\left|u-u_{h}^{\mathrm{MS}}\right|_{H^{1}(\Omega), \alpha} \leq C h\left[h|f|_{H^{1 / 2}(\Omega)}^{2}+\|f\|_{L_{2}(\Omega)}^{2}\right]^{1 / 2},
$$




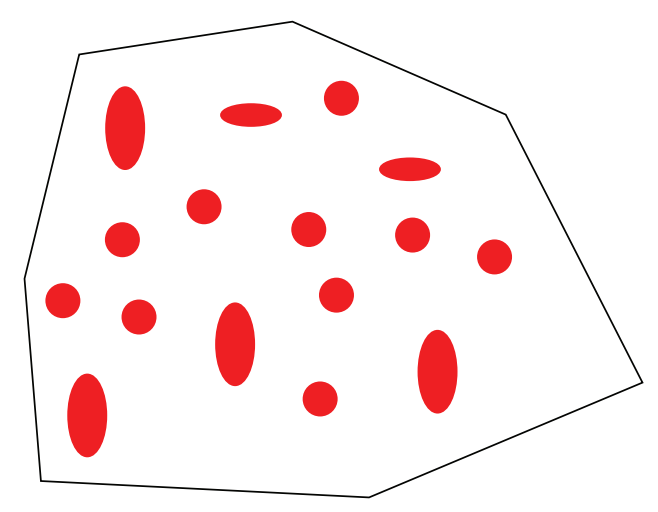

Figure 1. A domain with many inclusions

where

$$
|v|_{H^{1}(\Omega), \alpha}^{2}=a(v, v), \quad \text { with } \quad a(v, w)=\int_{\Omega} \alpha \nabla v \cdot \nabla w,
$$

and the constant $C$ is independent of $h$ and of the contrast parameter $\widehat{\alpha}$. This should be compared to the best result of $\mathcal{O}\left(h^{1 / 2-\varepsilon}\right.$ ) (with a hidden constant which generally depends on the contrast) for standard finite element methods on a mesh which does not resolve the interface. We also devise a non-standard duality argument which shows that

$$
\left\|u-u_{h}^{\mathrm{MS}}\right\|_{L_{2}(\Omega)} \leq C h^{2}\left[h|f|_{H^{1 / 2}(\Omega)}^{2}+\|f\|_{L_{2}(\Omega)}^{2}\right]^{1 / 2} .
$$

The price to pay for this improved convergence rate is the solution of subgrid problems on elements which straddle the interface and a slightly worse dependence than normal on the data $f$ on the right-hand sides of (1.7) and (1.8). The local subgrid problems can be done as a preprocessing step before the solution of the global finite element problem. The accuracy needed for these subgrid problems is investigated numerically in 4

The multiscale finite element method (in which basis functions are computed by solving local homogeneous PDEs subject to special boundary conditions) has a large literature. The primitive form of this method can be traced back to the early work of Babuška, Caloz and Osborn [5, 4, who introduced special basis functions for 1D elliptic problems with rough coefficients.

In 19 , Hou and $\mathrm{Wu}$ developed the multiscale finite element method for multidimensional problems with multiscale coefficients. The main idea of the method was to incorporate the small-scale features in the underlying physical problem into the finite element bases locally. Within each coarse grid block, one can construct the multiscale basis by solving the leading order governing equation locally. The small scales then interact with the large scales through the variational formulation of the finite element method. Hou and $\mathrm{Wu}[19]$ also identified a key issue in multiscale methods, that is, the microscopic boundary condition which connects the small scale bases to the macroscopic solution. They further performed a convergence analysis to reveal a resonance error introduced by the microscopic boundary condition [19, 20]. An over-sampling technique was proposed to effectively reduce the resonance error 19. The multiscale bases are strongly localized and adaptive. In many cases, the 
multiscale bases can be pre-computed and can be used repeatedly in subsequent computations with different source terms and different boundary conditions. In some situations the basis functions can be updated adaptively. This leads to a significant computational saving in upscaling the two-phase flows where the elliptic (pressure) equation needs to be solved many times dynamically. Another attractive feature of the method is the ability to both coarsen (upscale) a fine grid solution and to reconstruct (downscale) the fine grid solution from a coarse grid solution by using the multiscale bases. This property is very attractive in many engineering applications.

Most of the convergence analysis of multiscale finite element methods is for the periodic homogenisation case where, in (1.2), $\alpha(x)=\alpha(x / \varepsilon)$, with $\varepsilon$ a small parameter and $\alpha$ a smooth positive-valued periodic function on a unit cell $Y$, and the analysis is geared to obtaining optimal convergence, robust with respect to the "oscillation parameter" $\varepsilon \rightarrow 0$ (e.g. [19, 20]). However, the method itself is quite general and has been applied to non-periodic cases with considerable success. For example in 19] one finds an application to (1.2) for the case when $\alpha$ is a realisation of a random field, both in the isotropic and anisotropic cases and with highly contrasting media. We also refer to a recent book by Efendiev and Hou 12 for more discussions on the theory and applications of multiscale finite element methods.

The convergence analysis of the new multiscale finite element method devised in this paper makes no appeal to homogenisation theory but nevertheless explains why "multiscale"-type basis construction can be beneficial in more general situations. It turns out that the new interior boundary conditions obtained in the present work are a genuine generalisation of the "oscillatory" boundary conditions of [19], in the sense that the two coincide if and only if the interfaces intersect the element edges orthogonally. Some of the arguments used in this paper have already been developed in the context of domain decomposition methods in [15, 16, 36.

To our knowledge the dependence of the accuracy of numerical methods for elliptic interface problems on coefficient contrast has not been previously analysed, even though such high contrast problems are ubiquitous in porous media problems, especially in geophysical and oil recovery applications. We note, however, that there is substantial literature on the performance of iterative methods (see, e.g. [15] and the references therein) and on the analysis of a posterior error estimates (see, e.g. [7, 2, 44]) for such high contrast problems, but this literature does not address the issues considered in the present paper.

In the following section, 92 , we explain the main idea behind our method and illustrate the analysis in the special case when the coarse mesh can be drawn to enclose each of the inclusions $\Omega_{i}: i=1, \ldots, m$. The main results of the paper are obtained in 33, where we construct the multiscale basis functions in the case when the coefficient interface intersects a typical triangular coarse mesh element. Here we present a detailed analysis, obtaining the estimates (1.7) and (1.8) under certain technical assumptions. Numerical experiments which illustrate these results are provided in 84 . Two new technical results which are crucial to the analysis are included as appendices: The first is a scaled version of the trace theorem on a triangular annulus, while the second is a regularity theorem (due to N. Babych, I. Kamotski and V.P. Smyshlyaev of the University of Bath) for the exact solution of (1.2) in the high-contrast case. These should be of independent interest. 
Before we embark on $₫ 2$, we first discuss relevant existing literature on interface problems of the form (1.1). The approach that is closest to ours is the immersed finite element (IFE) method of $\mathrm{Li}$, Lin and $\mathrm{Wu}$ [28]. This method considered uniform triangular grids and approximated the interface by a straight line segment when it intersects a coarse grid element. By matching the jump condition, they created a special basis function for elements which are cut through by the interface and proved a second order convergence rate in the $L_{2}$ norm and a first order convergence rate in the $H^{1}$ semi-norm. On the other hand, the constants in their error estimate depend strongly on the contrast of the coefficient. It turns out that when the interface intersects an element in a straight line, our new method coincides with the method of [28]. Thus our results show in fact robustness with respect to the contrast for the IFE method and hence help explain the rather good numerical experiments in 28]. The connections between our method and that of [28] are further discussed in 3.2

Much earlier, Babuška [3] studied the convergence of methods based on a minimization problem equivalent to (1.1) in which the boundary and jump condition were incorporated in the cost functions. There are many subsequent works on such penalty methods, for example, Barrett and Elliott 6]. Another relevant work is due to Chen and Zou 9 who approximated the smooth interface by a polygon and used classical finite element methods to solve both elliptic and parabolic interface equations. The disadvantage of this approach is that the mesh must align with the interface. Plum and Wieners [39] studied interface problems with piecewise constant coefficients and proved (under certain assumptions) optimal a priori estimates which are independent of the coefficients for standard finite element methods with meshes resolving the interface. Related results for discontinuous Galerkin methods were given by Dryja in [10]. Unfitted high order finite element methods were recently studied by Li et al. in [25], and error estimates which are explicit in both the order of the elements and the error in the boundary approximation were proved.

There has been a great deal of effort in developing accurate and efficient finite difference methods for the interface problem. Among them, the Immersed Boundary Method (IBM) was developed by Peskin 37 for studying the motion of one or more massless, elastic surfaces immersed in an incompressible, viscous fluid, particularly in biofluid dynamics problems where complex geometries and immersed elastic membranes are present. The IBM method employs a uniform Eulerian grid over the entire domain to describe the velocity field of the fluid and a Lagrangian description for the immersed elastic structure. The interaction between the fluid and the structure is expressed in terms of the spreading and interpolation operations by use of smoothed delta functions. We refer to [38] for an extensive review of this method and its various applications. Motivated by Peskin's method, Unverdi and Tryggvason have developed a highly successful front tracking method to study viscous incompressible multiphase flows [42].

Another related work is the Immersed Interface Method (IIM) for elliptic interface problems developed by LeVeque and Li [24. The main idea of the IIM method is to use the jump condition across the interface to modify the finite difference approximation near the interface. When this is done properly, one can achieve a second order discretization. The IIM method can also be applied to the moving interface problem [18] and to the irregular domain problem [11]. Several extensions and improvements can be found in the references [1, 26, 27. 
An important development of interface capturing methods is the Ghost Fluid Method (GFM) [13 developed by Fedkiw, Merriman, Aslam and Osher. The GFM incorporated the interface jump condition into the finite difference discretization in a clever way which can be implemented efficiently. The GFM has been applied to capture discontinuities in multimedium compressible flow [22] and strong shock impacting problems [23], with considerable success. The GFM has been generalized to the elliptic interface problem in 30, and its convergence property has been analyzed in 31. Related works include [45, 8. We remark that there has been little progress in rigorous convergence theory for finite difference methods for interface problems. By contrast, the present paper proves the robust convergence of our proposed method.

\section{THE KEY IDEA AND A SIMPLE APPLICATION}

2.1. The key idea. For any measurable subset $D \subset \Omega$, define the local version of $a$ :

$$
a_{D}(v, w)=\int_{D} \alpha \nabla v \cdot \nabla w .
$$

For a suitable index set $\mathscr{I}_{h}(D)$, let $\mathcal{N}_{h}(D)=\left\{x_{p}: p \in \mathscr{I}_{h}(D)\right\}$ denote the nodes of the mesh $\mathcal{T}_{h}$ which lie in $D$. For any triangular element $\tau \in \mathcal{T}_{h}$ (assumed to contain its boundary), $\mathcal{N}_{h}(\tau)=\left\{x_{p}: p \in \mathscr{I}_{h}(\tau)\right\}$ is the set containing the three nodes of $\tau$. For each $p \in \mathscr{I}_{h}(\tau)$ we shall construct nodal basis functions $\Phi_{p}^{\mathrm{MS}}$, whose restriction $\Phi_{p, \tau}^{\mathrm{MS}}$ to each $\tau \in \mathcal{T}^{H}$ is required to solve the "subgrid problem":

$$
a_{\tau}\left(\Phi_{p, \tau}^{\mathrm{MS}}, v\right)=0 \quad \text { for all } \quad v \in H_{0}^{1}(\tau)
$$

together with a suitable boundary condition:

$$
\Phi_{p, \tau}^{\mathrm{MS}}=\phi_{p, \tau} \quad \text { on } \quad \partial \tau, \quad \text { with } \quad \phi_{p, \tau}\left(x_{q}\right)=\delta_{p, q} \quad \text { for all } \quad p, q \in \mathscr{I}_{h}(\tau),
$$

where $\phi_{p, \tau} \in \mathcal{C}(\partial \tau)$ and

$$
\sum_{p \in \mathscr{I}_{h}(\tau)} \phi_{p, \tau}=1 \quad \text { on } \quad \partial \tau .
$$

In general for each $p \in \mathscr{I}_{h}(\bar{\Omega})$ the boundary data in (2.2) has to be prescribed and the local problems (2.1) may have to be solved (e.g. on a subgrid). (We will see that there is a bounded number of these local problems for each $p$, independent of the coarse mesh diameter.) However, if $\alpha$ is constant on $\tau$, then the boundary condition will be chosen so that $\Phi_{p, \tau}^{\mathrm{MS}}$ is just the linear hat function on $\tau$ centred at $x_{p}$. Observe that under conditions (2.1), (2.2) and (2.3),

$$
\sum_{p \in \mathscr{I}_{h}(\tau)} \Phi_{p, \tau}^{\mathrm{MS}}=1 \quad \text { on } \quad \tau .
$$

From the basis functions we construct the nodal interpolation operator: $\mathcal{I}_{h}^{\mathrm{MS}} v=$ $\sum_{p \in \mathscr{I}_{h}(\bar{\Omega})} v\left(x_{p}\right) \Phi_{p}^{\mathrm{MS}}$, which is defined for all $v \in C(\bar{\Omega})$. Note that from (2.4) it follows that

$$
\mathcal{I}_{h}^{\mathrm{MS}} 1=1 \quad \text { on } \bar{\Omega} .
$$

The basis functions $\Phi_{p}^{\mathrm{MS}}$ which we shall construct will be continuous across element edges and will vanish on $\partial \Omega$, so that

$$
\mathcal{V}_{h}^{\mathrm{MS}}:=\operatorname{span}\left\{\Phi_{p}^{\mathrm{MS}}: p \in \mathscr{I}_{h}(\Omega)\right\} \subseteq H_{0}^{1}(\Omega),
$$


i.e. the method is conforming. Then we define the finite element solution $u_{h}^{\mathrm{MS}} \in$ $\mathcal{V}_{h}^{\mathrm{MS}}$ of (1.2) by requiring that $a\left(u_{h}^{\mathrm{MS}}, v_{h}^{\mathrm{MS}}\right)=\left(f, v_{h}^{\mathrm{MS}}\right)_{L_{2}(\Omega)}$, for all $v_{h}^{\mathrm{MS}} \in \mathcal{V}_{h}^{\mathrm{MS}}$, which gives us the usual optimality estimate:

$$
\left|u-u_{h}^{\mathrm{MS}}\right|_{H^{1}(\Omega), \alpha} \leq\left|u-\mathcal{I}_{h}^{\mathrm{MS}} u\right|_{H^{1}(\Omega), \alpha} .
$$

Now, to estimate the right-hand side of (2.7), we note that, since the basis functions satisfy the homogeneous equation (2.1), and since $u$ solves (1.2), the local interpolation error $E_{h}^{\mathrm{MS}}:=u-\mathcal{I}_{h}^{\mathrm{MS}} u$ satisfies, for all $\tau \in \mathcal{T}_{h}$,

$$
a_{\tau}\left(E_{h}^{\mathrm{MS}}, v\right)=(f, v)_{L_{2}(\tau)} \quad \text { for all } \quad v \in H_{0}^{1}(\tau) .
$$

An estimate for $\left|E_{h}^{\mathrm{MS}}\right|_{H^{1}(\Omega), \alpha}$, suitable for inserting in the right-hand side of (2.7), can be obtained from the following lemma.

Lemma 2.1. Suppose $D$ is a Lipschitz subdomain of $\Omega$ and suppose that $\phi \in H^{1}(D)$ satisfies

$$
a_{D}(\phi, v)=(f, v)_{L_{2}(D)} \quad \text { for all } \quad v \in H_{0}^{1}(D) .
$$

Then for any $\tilde{\phi} \in H^{1}(D)$ such that the trace of $\tilde{\phi}-\phi$ vanishes on $\partial D$,

$$
|\phi|_{H^{1}(D), \alpha} \leq|\tilde{\phi}|_{H^{1}(D), \alpha}+C \operatorname{diam}(D)\|f\|_{L_{2}(D)},
$$

where $C$ is independent of $\phi, \tilde{\phi}$, the diameter of $D$ and $\widehat{\alpha}$.

Proof. Let $\phi^{*}$ be the unique solution of the problem

$$
a_{D}\left(\phi^{*}, v\right)=0 \text { for all } v \in H_{0}^{1}(D),
$$

such that the trace of $\phi^{*}-\phi$ vanishes on $\partial D$. Then $\phi-\phi^{*} \in H_{0}^{1}(D)$ and, by subtracting (2.10) from (2.9), $a_{D}\left(\phi-\phi^{*}, v\right)=(f, v)_{L_{2}(D)}$ for all $v \in H_{0}^{1}(D)$. Then

$$
\begin{aligned}
\left|\phi-\phi^{*}\right|_{H^{1}(D), \alpha}^{2} & =a_{D}\left(\phi-\phi^{*}, \phi-\phi^{*}\right)=a_{D}\left(\phi, \phi-\phi^{*}\right)=\left(f, \phi-\phi^{*}\right)_{L_{2}(D)} \\
& \leq\|f\|_{L_{2}(D)}\left\|\phi-\phi^{*}\right\|_{L_{2}(D)} \leq C \operatorname{diam}(D)\|f\|_{L_{2}(D)}\left|\phi-\phi^{*}\right|_{H^{1}(D), \alpha},
\end{aligned}
$$

where in the last step we used the Poincaré-Friedrichs inequality and also $\widehat{\alpha} \geq 1$. Hence

$$
|\phi|_{H^{1}(D), \alpha} \leq\left|\phi^{*}\right|_{H^{1}(D), \alpha}+C \operatorname{diam}(D)\|f\|_{L_{2}(D)} .
$$

On the other hand, (2.10) implies the minimality of the energy norm of $\phi^{*}$, i.e. $\left|\phi^{*}\right|_{H^{1}(D), \alpha} \leq|\tilde{\phi}|_{H^{1}(D), \alpha}$ for all $\tilde{\phi}$ satisfying the same boundary conditions as $\phi$, and the result follows.

Recalling (2.8) and using Lemma 2.1 to bound the right-hand side of (2.7), we then obtain

Theorem 2.2.

$$
\left|E_{h}^{\mathrm{MS}}\right|_{H^{1}(\tau), \alpha} \leq\left|\widetilde{E}_{h}^{\mathrm{MS}}\right|_{H^{1}(\tau), \alpha}+C h_{\tau}\|f\|_{L_{2}(\tau)}
$$

and

$$
\left|u-u_{h}^{\mathrm{MS}}\right|_{H^{1}(\Omega), \alpha} \leq C\left[\sum_{\tau \in \mathcal{T}_{h}}\left(\left|\widetilde{E}_{h}^{\mathrm{MS}}\right|_{H^{1}(\tau), \alpha}^{2}+h_{\tau}^{2}\|f\|_{L_{2}(\tau)}^{2}\right)\right]^{1 / 2},
$$

where $\widetilde{E}_{h}^{\mathrm{MS}}$ is any function whose trace coincides with the trace of $E_{h}^{\mathrm{MS}}$ on $\partial \tau$ and $C$ is a generic constant independent of $\mathcal{T}_{h}, f, u$ and $\alpha$. 
Note that, although simple, Theorem 2.2 represents a genuine extension of standard estimates. For example, if $\alpha$ is constant on each $\tau \in \mathcal{T}_{h}$, then the multiscale basis functions coincide with linear basis functions. Setting $\widetilde{E}_{h}^{\mathrm{MS}}=E_{h}^{\mathrm{MS}}$ (which now equals the error in linear interpolation) leads to

$$
\left|u-u_{h}^{\mathrm{MS}}\right|_{H^{1}(\Omega), \alpha} \leq C h\left[\sum_{\tau \in \mathcal{T}_{h}} \alpha_{\tau}|u|_{H^{2}(\tau)}^{2}+\|f\|_{L_{2}(\Omega)}^{2}\right]^{1 / 2},
$$

which yields the usual $\mathcal{O}(h)$ estimate in the energy norm with (a coefficient dependent) asymptotic constant. However, this also demonstrates the possibility that when $\alpha_{\tau}$ is large, small $|u|_{H^{2}(\tau)}^{2}$ could provide better estimates with respect to $\alpha$. Our regularity theory (in the Appendix) tells us that this is exactly what does happen. However, a deeper use of Theorem 2.2 may be envisaged when $\alpha$ varies within an element. Then it turns out to be possible (although not trivial) to define the boundary condition (2.2) in such a way that $\sum_{\tau \in \mathcal{T}_{h}}\left|\widetilde{E}_{h}^{\mathrm{MS}}\right|_{H^{1}(\tau), \alpha}^{2} \leq C h^{2}$, with constant $C$ independent of $\alpha$. We explain how this comes about in the context of a special case in the next subsection. Then in $\$ 3$ we extend to the more difficult case where the interface cuts through a mesh element.

From now on we shall make use of the following notational conventions.

Notation 2.3. We shall write $g_{1} \lesssim g_{2}$ when there exists a constant $C$ which is independent of $u, f, h, \alpha$ such that $g_{1} \leq C g_{2}$. Similarly, $g_{1} \sim g_{2}$ means $g_{1} \lesssim g_{2}$ and $g_{2} \lesssim g_{1}$.

Notation 2.4. For any suitably smooth function $\phi$ defined on $\tau \in \mathcal{T}_{h}$ and any edge $e$ of $\tau$, we define $D_{e}^{s} \phi$ for $s \geq 1$ to be the derivative of $\phi$ of order $s$ along $e$.

2.2. A simple application. In this subsection we assume that each of the inclusions $\Omega_{i}$ are enclosed inside elements of $\mathcal{T}_{h}$. More precisely, for any $\tau \in \mathcal{T}_{h}$ and any sufficiently small $\varepsilon>0$, let us define $\tau^{\varepsilon}=\{x \in \tau$ : $\operatorname{dist}(x, \partial \tau) \leq \varepsilon\}$. Then, for each $i=1, \ldots, m$, we assume that there exists $\tau_{i} \in \mathcal{T}_{h}$ and $\varepsilon_{i}>0$ such that

$$
\Omega_{i} \subseteq \tau_{i} \backslash \tau_{i}^{\varepsilon_{i}} .
$$

That is the boundary of $\Omega_{i}$ lies at least a distance $\varepsilon_{i}$ from the boundary of $\tau_{i}$. (Note that any element could contain more than one inclusion.) Our estimates will depend on the following measure of the relative size of $\varepsilon_{i}$ :

$$
\delta:=\max _{i=1, \ldots, m} \frac{h_{\tau_{i}}}{\varepsilon_{i}} .
$$

Note that $\delta \geq 1$. Then we have the following theorem:

Theorem 2.5. Suppose the boundary condition in (2.2) is chosen to be linear on the boundary of each element $\tau \in \mathcal{T}_{h}$. Then, for $f \in H^{1 / 2}(\Omega)$,

$$
\begin{aligned}
& \text { (i) } \quad\left|u-u_{h}^{\mathrm{MS}}\right|_{H^{1}(\Omega), \alpha} \lesssim \delta^{3} h\left[h|f|_{H^{1 / 2}(\Omega)}^{2}+\|f\|_{L_{2}(\Omega)}^{2}\right]^{1 / 2}, \\
& \text { (ii) } \quad\left\|u-u_{h}^{\mathrm{MS}}\right\|_{L_{2}(\Omega)} \lesssim \delta^{6} h^{2}\left[h|f|_{H^{1 / 2}(\Omega)}^{2}+\|f\|_{L_{2}(\Omega)}^{2}\right]^{1 / 2} .
\end{aligned}
$$

Proof. We give the proof in Case I (see (1.5)). That means $\alpha_{i} \geq \widehat{\alpha} \rightarrow \infty$ for $i=1, \ldots, m$ and $\alpha_{0}=1$. The proof in Case II is dealt with in Remark 2.6.

First consider any element $\tau_{i} \in \mathcal{T}_{h}$ which contains an inclusion $\Omega_{i}$. We construct $\widetilde{E}_{h}^{\mathrm{MS}}$ on $\tau_{i}$ by first defining it to be zero on $\tau_{i} \backslash \tau_{i}^{\varepsilon_{i}}$, equal to $E_{h}^{\mathrm{MS}}$ on $\partial \tau_{i}$, and then 
extending into $\tau_{i}^{\varepsilon_{i}}$, using Lemma A.1 of Appendix A (scaled to $\tau_{i}$ ). By combining this with standard estimates for linear approximation in 1D, we obtain

$$
\begin{aligned}
\left|\widetilde{E}_{h}^{\mathrm{MS}}\right|_{H^{1}\left(\tau_{i}\right), \alpha}^{2} & =\left|\widetilde{E}_{h}^{\mathrm{MS}}\right|_{H^{1}\left(\tau_{i}^{\varepsilon_{i}}\right)}^{2} \\
& \lesssim\left(\frac{h_{\tau_{i}}}{\varepsilon_{i}}\right)^{2} h_{\tau_{i}}^{-1}\left\|E_{h}^{\mathrm{MS}}\right\|_{L_{2}\left(\partial \tau_{i}\right)}^{2}+\left(\frac{h_{\tau_{i}}}{\varepsilon_{i}}\right)^{2} h_{\tau_{i}}\left|E_{h}^{\mathrm{MS}}\right|_{H^{1}\left(\partial \tau_{i}\right)}^{2} \\
& \lesssim \delta^{2} h_{\tau_{i}}^{3} \sum_{e \in \mathcal{E}\left(\tau_{i}\right)}\left\|D_{e}^{2} u\right\|_{L_{2}(e)}^{2},
\end{aligned}
$$

where $\mathcal{E}\left(\tau_{i}\right)$ denotes the edges of $\tau$. On the other hand if $\tau \in \mathcal{T}_{h}$ contains no inclusion, then with $\widetilde{E}_{h}^{\mathrm{MS}}:=E_{h}^{\mathrm{MS}}$ it is easy to show that this estimate remains true with $\delta$ replaced by 1 ; thus

$$
\left|\widetilde{E}_{h}^{\mathrm{MS}}\right|_{H^{1}(\tau), \alpha}^{2} \lesssim \delta^{2} h_{\tau}^{3} \sum_{e \in \mathcal{E}(\tau)}\left\|D_{e}^{2} u\right\|_{L_{2}(e)}^{2} \quad \text { for all } \quad \tau \in \mathcal{T}_{h} .
$$

By recalling (2.12), we can now see that (2.18) would allow us to estimate $\left|u-u_{h}^{\mathrm{MS}}\right|_{H^{1}(\Omega), \alpha}$ in terms of the data $f$ and certain derivatives of the solution $u$ along edges which (in this case) lie entirely in $\Omega_{0}$. In order to be able to prove robustness to the contrast $\widehat{\alpha}$, we now estimate these edge derivatives in terms of Sobolev norms of $u$ in $\Omega_{0}$, which we can in turn estimate independently of $\widehat{\alpha}$, using the regularity theory in the Appendix. The required technical argument, which we now give, leads to (2.29).

First we recall the trace theorem for polygons (e.g. [17, Theorem 1.5.2.1]), which, after scaling to any element $\tau \in \mathcal{T}_{h}$, reads

$$
|v|_{H^{1}(e)}^{2} \lesssim h_{\tau}^{-3}\|v\|_{L_{2}(\tau)}^{2}+h_{\tau}^{-1}|v|_{H^{1}(\tau)}^{2}+|v|_{H^{3 / 2}(\tau)}^{2} \quad \text { for all } \quad v \in H^{3 / 2}(\tau) \text {. }
$$

Moreover, replacing $v$ by $v-\gamma$ where $\gamma$ is an arbitrary constant and then invoking the Poincaré inequality on $\tau$, we easily obtain the simpler estimate:

$$
|v|_{H^{1}(e)}^{2} \lesssim h_{\tau}^{-1}|v|_{H^{1}(\tau)}^{2}+|v|_{H^{3 / 2}(\tau)}^{2} \quad \text { for all } \quad v \in H^{3 / 2}(\tau) \quad \text { and all } \quad \tau \in \mathcal{T}_{h} .
$$

Now return to the case where $\tau=\tau_{i}$, an element which contains an inclusion $\Omega_{i}$. Choose $\phi$ to be a $\mathcal{C}^{\infty}$ cut-off function which vanishes on $\tau_{i} \backslash \tau_{i}^{\varepsilon_{i}}$, has the value 1 on $\partial \tau_{i}$ and satisfies $\left\|D^{\beta} \phi\right\|_{L^{\infty}\left(\tau_{i}\right)} \lesssim \varepsilon_{i}^{-|\beta|}$ for all multi-indices $\beta$. Then choose any edge $e$ of $\tau_{i}$ and any constant $\gamma$. Using the fact that $\phi \gamma$ is constant on $e$ and then (2.19), we obtain

$\left\|D_{e}^{2} u\right\|_{L_{2}(e)}^{2}=\left|\phi\left(D_{e} u-\gamma\right)\right|_{H^{1}(e)}^{2} \lesssim h_{\tau_{i}}^{-1}\left|\phi\left(D_{e} u-\gamma\right)\right|_{H^{1}\left(\tau_{i}\right)}^{2}+\left|\phi\left(D_{e} u-\gamma\right)\right|_{H^{3 / 2}\left(\tau_{i}\right)}^{2}$.

To estimate the right-hand side, we first recall the Poincaré inequality for an annulus (Lemma A.2) and scale it to $\tau_{i}$ in order to obtain a constant $\gamma \in \mathbb{R}$ such that

$$
\|v-\gamma\|_{L_{2}\left(\tau_{i}^{\varepsilon_{i}}\right)} \lesssim h_{\tau_{i}}|v|_{H^{1}\left(\tau_{i}^{\varepsilon_{i}}\right)} \text { for all } v \in H^{1}\left(\tau_{i}^{\varepsilon_{i}}\right),
$$

with a hidden constant independent of both $h_{\tau_{i}}$ and $\varepsilon_{i}$.

Now, to estimate the first term on the right-hand side of (2.20), use the above estimates for the derivatives of $\phi$ and then (2.21) in order to obtain

$$
\begin{aligned}
h_{\tau_{i}}^{-1}\left|\phi\left(D_{e} u-\gamma\right)\right|_{H^{1}\left(\tau_{i}\right)}^{2} & \lesssim h_{\tau_{i}}^{-1} \varepsilon_{\tau_{i}}^{-2}\left\|D_{e} u-\gamma\right\|_{L_{2}\left(\tau_{i}^{\varepsilon_{i}}\right)}^{2}+h_{\tau_{i}}^{-1}|u|_{H^{2}\left(\tau_{i}^{\varepsilon_{i}}\right)}^{2} \\
& \lesssim h_{\tau_{i}}^{-1} \delta^{2}|u|_{H^{2}\left(\tau_{i}^{\varepsilon_{i}}\right)}^{2} .
\end{aligned}
$$


Estimation of the second term on the right-hand side of (2.20) is slightly more involved. For any multi-index $\beta$ of order 1 , we have

$\left|\phi\left(D_{e} u-\gamma\right)\right|_{H^{3 / 2}\left(\tau_{i}\right)}^{2} \lesssim \sum_{|\beta|=1}\left|\left(D^{\beta} \phi\right)\left(D_{e} u-\gamma\right)\right|_{H^{1 / 2}\left(\tau_{i}^{\varepsilon_{i}}\right)}^{2}+\sum_{|\beta|=1}\left|\phi D^{\beta} D_{e} u\right|_{H^{1 / 2}\left(\tau_{i}^{\varepsilon_{i}}\right)}^{2}$.

Now for all $\psi \in \mathcal{C}^{\infty}\left(\tau_{i}^{\varepsilon_{i}}\right)$ and $v \in H^{1 / 2}\left(\tau_{i}^{\varepsilon_{i}}\right)$, elementary arguments show

$$
|\psi v|_{H^{1 / 2}\left(\tau_{i}^{\varepsilon_{i}}\right)}^{2} \leq\|\psi\|_{L_{\infty}\left(\tau_{i}^{\varepsilon_{i}}\right)}^{2}|v|_{H^{1 / 2}\left(\tau_{i}^{\varepsilon_{i}}\right)}^{2}+h_{\tau_{i}}\|\nabla \psi\|_{L_{\infty}\left(\tau_{i}^{\varepsilon_{i}}\right)}^{2}\|v\|_{L_{2}\left(\tau_{i}^{\varepsilon_{i}}\right)}^{2} .
$$

Hence, the first term on the right-hand side of (2.23) is estimated by

$$
\left|\left(D^{\beta} \phi\right)\left(D_{e} u-\gamma\right)\right|_{H^{1 / 2}\left(\tau_{i}^{\varepsilon_{i}}\right)}^{2} \lesssim \varepsilon_{\tau_{i}}^{-2}\left|D_{e} u-\gamma\right|_{H^{1 / 2}\left(\tau_{i}^{\varepsilon_{i}}\right)}^{2}+\varepsilon_{\tau_{i}}^{-4} h_{\tau_{i}}\left\|D_{e} u-\gamma\right\|_{L_{2}\left(\tau_{i}^{\varepsilon_{i}}\right)}^{2} .
$$

Now interpolating (2.21) with the corresponding estimate for $\left\|D_{e} u-\gamma\right\|_{H^{1}\left(\tau_{i}^{\varepsilon_{i}}\right)}$ we obtain

$$
\left|D_{e} u-\gamma\right|_{H^{1 / 2}\left(\tau_{i}^{\varepsilon_{i}}\right)} \lesssim h_{\tau_{i}}^{1 / 2}\left|D_{e} u\right|_{H^{1}\left(\tau_{i}^{\varepsilon_{i}}\right)} .
$$

Combining (2.21) and (2.26) with (2.25), we have

$$
\left|\left(D^{\beta} \phi\right)\left(D_{e} u-\gamma\right)\right|_{H^{1 / 2}\left(\tau_{i}^{\varepsilon_{i}}\right)}^{2} \lesssim\left(\varepsilon_{\tau_{i}}^{-2} h_{\tau_{i}}+\varepsilon_{\tau_{i}}^{-4} h_{\tau_{i}}^{3}\right)|u|_{H^{2}\left(\tau_{i}^{\varepsilon_{i}}\right)}^{2} \lesssim h_{\tau_{i}}^{-1} \delta^{4}|u|_{H^{2}\left(\tau_{i}^{\varepsilon_{i}}\right)}^{2} .
$$

The second term on the right-hand side of (2.23) is also estimated by (2.24):

$\left|\phi D^{\beta} D_{e} u\right|_{H^{1 / 2}\left(\tau_{i}^{\varepsilon_{i}}\right)}^{2} \lesssim|u|_{H^{5 / 2}\left(\tau_{i}^{\varepsilon_{i}}\right)}^{2}+h_{\tau_{i}} \varepsilon_{\tau_{i}}^{-2}|u|_{H^{2}\left(\tau_{i}^{\varepsilon_{i}}\right)}^{2} \lesssim|u|_{H^{5 / 2}\left(\tau_{i}^{\varepsilon_{i}}\right)}^{2}+h_{\tau_{i}}^{-1} \delta^{2}|u|_{H^{2}\left(\tau_{i}^{\varepsilon_{i}}\right)}^{2}$.

Then by combining (2.27) and (2.28) with (2.23), we have

$$
\left|\phi\left(D_{e} u-\gamma\right)\right|_{H^{3 / 2}\left(\tau_{i}\right)}^{2} \lesssim h_{\tau_{i}}^{-1} \delta^{4}|u|_{H^{2}\left(\tau_{i}^{\varepsilon_{i}}\right)}^{2}+|u|_{H^{5 / 2}\left(\tau_{i}^{\varepsilon_{i}}\right)}^{2} .
$$

Combining this with (2.22) and (2.20), we have

$$
\left\|D_{e}^{2} u\right\|_{L_{2}(e)}^{2} \lesssim h_{\tau_{i}}^{-1} \delta^{4}|u|_{H^{2}\left(\tau_{i}^{\varepsilon_{i}}\right)}^{2}+|u|_{H^{5 / 2}\left(\tau_{i}^{\varepsilon_{i}}\right)}^{2} .
$$

By a direct application of (2.19), this estimate also holds (in fact with $\delta$ replaced by 1 ) when $\tau$ does not contain an inclusion, so that

$\left\|D_{e}^{2} u\right\|_{L_{2}(e)}^{2} \lesssim h_{\tau}^{-1} \delta^{4}|u|_{H^{2}\left(\tau \cap \Omega_{0}\right)}^{2}+|u|_{H^{5 / 2}\left(\tau \cap \Omega_{0}\right)}^{2} \quad$ for all $\quad e \in \mathcal{E}(\tau)$ and all $\tau \in \mathcal{T}_{h}$.

Combining this with (2.18) yields, for all $\tau \in \mathcal{T}_{h}$,

$$
\left|\widetilde{E}_{h}^{\mathrm{MS}}\right|_{H^{1}(\tau), \alpha}^{2} \lesssim \delta^{2} h_{\tau}^{2}\left[\delta^{4}|u|_{H^{2}\left(\tau \cap \Omega_{0}\right)}^{2}+h_{\tau}|u|_{H^{5 / 2}\left(\tau \cap \Omega_{0}\right)}^{2}\right] .
$$

Combining this with (2.12) and employing the regularity theory from the Appendix yields the result (i).

To obtain (ii), we use a non-standard variant of the usual duality argument. Let $w \in H_{0}^{1}(\Omega)$ be the solution of

$$
a(w, v)=\left(u-u_{h}^{\mathrm{MS}}, v\right)_{L_{2}(\Omega)} \quad \text { for all } \quad v \in H_{0}^{1}(\Omega),
$$

and let $w_{h}^{\mathrm{MS}} \in \mathcal{V}_{h}^{\mathrm{MS}}$ satisfy

$$
a\left(w_{h}^{\mathrm{MS}}, v_{h}^{\mathrm{MS}}\right)=\left(u-u_{h}^{\mathrm{MS}}, v_{h}^{\mathrm{MS}}\right)_{L_{2}(\Omega)} \quad \text { for all } \quad v_{h}^{\mathrm{MS}} \in \mathcal{V}_{h}^{\mathrm{MS}} .
$$


By applying (2.16), the interpolation theorem for $H^{1 / 2}(\Omega)$ and finally the arithmeticgeometric mean inequality $\left(a b \leq a^{2} / 2+b^{2} / 2\right)$ we obtain

$$
\begin{aligned}
\left|w-w_{h}^{\mathrm{MS}}\right|_{H^{1}(\Omega), \alpha} & \lesssim \delta^{3} h\left[h\left|u-u_{h}^{\mathrm{MS}}\right|_{H^{1 / 2}(\Omega)}^{2}+\left\|u-u_{h}^{\mathrm{MS}}\right\|_{L_{2}(\Omega)}^{2}\right]^{1 / 2} \\
& \lesssim \delta^{3} h\left[h\left|u-u_{h}^{\mathrm{MS}}\right|_{H^{1}(\Omega)}\left\|u-u_{h}^{\mathrm{MS}}\right\|_{L_{2}(\Omega)}+\left\|u-u_{h}^{\mathrm{MS}}\right\|_{L_{2}(\Omega)}^{2}\right]^{1 / 2} \\
& \lesssim \delta^{3} h\left[h^{2}\left|u-u_{h}^{\mathrm{MS}}\right|_{H^{1}(\Omega)}^{2}+\left\|u-u_{h}^{\mathrm{MS}}\right\|_{L_{2}(\Omega)}^{2}\right]^{1 / 2} \\
& \lesssim \delta^{3} h\left[h\left|u-u_{h}^{\mathrm{MS}}\right|_{H^{1}(\Omega)}+\left\|u-u_{h}^{\mathrm{MS}}\right\|_{L_{2}(\Omega)}\right] .
\end{aligned}
$$

Hence, taking $v=u-u_{h}^{\mathrm{MS}}$ in (2.30), we get

$$
\begin{aligned}
\left\|u-u_{h}^{\mathrm{MS}}\right\|_{L_{2}(\Omega)}^{2} & =a\left(w, u-u_{h}^{\mathrm{MS}}\right)=a\left(w-w_{h}^{\mathrm{MS}}, u-u_{h}^{\mathrm{MS}}\right) \\
& \leq\left|w-w_{h}^{\mathrm{MS}}\right|_{H^{1}(\Omega), \alpha}\left|u-u_{h}^{\mathrm{MS}}\right|_{H^{1}(\Omega), \alpha},
\end{aligned}
$$

and combining this with (2.32) (and recalling $\alpha \geq 1$ ), we have

$$
\left\|u-u_{h}^{\mathrm{MS}}\right\|_{L_{2}(\Omega)}^{2} \leq C \delta^{3} h\left[h\left|u-u_{h}^{\mathrm{MS}}\right|_{H^{1}(\Omega), \alpha}^{2}+\left|u-u_{h}^{\mathrm{MS}}\right|_{H^{1}(\Omega), \alpha}\left\|u-u_{h}^{\mathrm{MS}}\right\|_{L_{2}(\Omega)}\right]
$$

for some constant $C>0$. Now again by the arithmetic-geometric mean inequality we have

$$
C \delta^{3} h\left|u-u_{h}^{\mathrm{MS}}\right|_{H^{1}(\Omega), \alpha}\left\|u-u_{h}^{\mathrm{MS}}\right\|_{L_{2}(\Omega)} \leq \frac{1}{2} C^{2} \delta^{6} h^{2}\left|u-u_{h}^{\mathrm{MS}}\right|_{H^{1}(\Omega), \alpha}^{2}+\frac{1}{2}\left\|u-u_{h}^{\mathrm{MS}}\right\|_{L_{2}(\Omega)}^{2},
$$

and substitution into (2.33) yields

$$
\begin{aligned}
\frac{1}{2}\left\|u-u_{h}^{\mathrm{MS}}\right\|_{L_{2}(\Omega)}^{2} & \leq C \delta^{3} h^{2}\left|u-u_{h}^{\mathrm{MS}}\right|_{H^{1}(\Omega), \alpha}^{2}+\frac{1}{2} C^{2} \delta^{6} h^{2}\left|u-u_{h}^{\mathrm{MS}}\right|_{H^{1}(\Omega), \alpha}^{2} \\
& =C \delta^{3}\left(1+(C / 2) \delta^{3}\right) h^{2}\left|u-u_{h}^{\mathrm{MS}}\right|_{H^{1}(\Omega), \alpha}^{2},
\end{aligned}
$$

which, combined with (2.16), leads to the desired result (2.17).

Remark 2.6. For Case II, we can use the same idea to prove

$$
\begin{aligned}
\text { (i) } \quad\left|u-u_{h}^{\mathrm{MS}}\right|_{H^{1}(\Omega), \alpha} & \lesssim \delta^{3} h\left[h|f|_{H^{1 / 2}(\Omega)}^{2}+\|f\|_{L_{2}(\Omega)}^{2}\right]^{1 / 2} \\
\text { (ii) } \quad\left\|u-u_{h}^{\mathrm{MS}}\right\|_{L_{2}(\Omega)} & \lesssim \delta^{6} h^{2}\left[h|f|_{H^{1 / 2}(\Omega)}^{2}+\|f\|_{L_{2}(\Omega)}^{2}\right]^{1 / 2} .
\end{aligned}
$$

We construct $\widetilde{E}_{h}^{\mathrm{MS}}$ as in the proof of Theorem 2.5 and notice that $\left|\widetilde{E}_{h}^{\mathrm{MS}}\right|_{H^{1}\left(\tau_{i}\right), \alpha}=$ $\widehat{\alpha}^{1 / 2}\left|\widetilde{E}_{h}^{\mathrm{MS}}\right|_{H^{1}\left(\tau_{i}\right)}$. The regularity result in Theorem B.1 leads to (2.34). The same duality argument gives (2.35).

The estimates above can also be extended to the inhomogeneous Dirichlet case. This is discussed in detail in the more general context in Remark 3.19.

\section{ERror ANALYSIS FOR ELEMENTS WHICH INTERSECT INCLUSIONS}

Now we want to consider the case where the interface may intersect with the boundaries of some of the mesh elements. Recalling Theorem 2.2 we have to show that for each element $\tau$ there is a boundary condition for the multiscale basis functions on $\partial \tau$ such that $E_{h}^{\mathrm{MS}}:=u-\mathcal{I}_{h}^{\mathrm{MS}} u$ has an extension $\widetilde{E}_{h}^{\mathrm{MS}}$ from $\partial \tau$ into $\tau$, with a suitably bounded energy. 
In 3.1 we present some qualitative properties of the exact solution $u$ of (1.2) in the generic case when the interface intersects two edges of $\tau$. These relate various derivatives of $u$ at the points of edge-interface intersection, plus controllable remainders. These relations motivate the interior boundary conditions for the multiscale basis functions which are presented in $\$ 3.2$. In particular, it is explained how the boundary conditions can be found by solving a $6 \times 6$ linear system for each element which intersects the interface. In Theorem 3.9 we estimate $E_{h}^{\mathrm{MS}}$ on each element boundary.

The interior error is then considered in $\$ 3.3$. First Lemma 3.15 uses the result of Theorem 3.9 to prove the existence of an extension $\widetilde{E}_{h}^{\mathrm{MS}}$ of $E_{h}^{\mathrm{MS}}$ with suitably bounded energy. This leads to Theorem 3.16, which proves a suitable error estimate for the energy norm of $E_{h}^{\mathrm{MS}}$ on each element $\tau$, making use of Theorem 2.2 and the estimate for the extension proved in Theorem 3.9

The procedure that we describe constructs interior boundary conditions elementby-element and does not naturally lead to conforming elements. However, conformity can be retained by local averaging. This is described in 3.4 where the main theorem, Theorem 3.18, is proved. An important observation (discussed in 33.2) is that in the special case when the interface intersects the element edges orthogonally, our boundary condition coincides with the "oscillatory boundary conditions" proposed in [19].

3.1. Properties of the exact solution. In this subsection we derive some properties of the exact solution of (1.2) in an element $\tau$ through which an intersection cuts. To reduce some technicalities in the theory we shall make the following geometrical assumption on elements which intersect the interface.

Assumption 3.1. When the interface $\Gamma$ intersects an element $\tau$, we shall assume that it subdivides $\tau$ into two parts. We label the vertices of $\tau$ as $x_{1}, x_{2}, x_{3}$ in such a way that $\Gamma$ intersects $\partial \tau$ at points which we denote by $y_{i}$ in the edges $\overline{x_{3} x_{i}}$ for $i=1,2$. We let $\beta$ denote the angle of $\tau$ subtended at $x_{3}$, and we let $\tau^{-}$denote the part where $\alpha \geq \widehat{\alpha}$ and $\tau^{+}$denote the part where $\alpha \leq K$ (see (1.5), (1.6)). A typical situation is depicted in Figure 2, Letting $r_{i}^{-}$and $r_{i}^{+}$denote, respectively, the length of the line segments $e_{i} \cap \tau^{-}$and $e_{i} \cap \tau^{+}$, we shall assume that there exist positive constants $0 \leq \underline{R}<\bar{R} \leq 1$ and $0<B<\pi$ such that, for all meshes (characterised by mesh parameter $h$ ),

$\underline{R} h_{\tau} \leq \min \left\{r_{i}^{-}, r_{i}^{+}\right\} \leq \max \left\{r_{i}^{-}, r_{i}^{+}\right\} \leq \bar{R} h_{\tau} \quad$ for $\quad i=1,2$ and $\quad \pi-B \geq \beta \geq B$.

For $i=1,2$ we define $\theta_{i} \in(-\pi / 2, \pi / 2)$ to be the unique angle such that

$$
\mathbf{e}_{i}=\cos \theta_{i} \mathbf{n}_{i}+\sin \theta_{i} \mathbf{t}_{i} .
$$

Then we also assume that $\Gamma$ is not tangential to either of the edges $e_{i}$, i.e.

$$
\left|\theta_{i}\right| \leq \pi / 2-T \text { for some } T>0 .
$$

The hidden constants in the estimates below may depend on $\beta, \underline{R}, \bar{R}, B$ and $T$.

In many cases where the element intersects the interface in a different way, we can always find a refinement to reduce to cases satisfying Assumption 3.1; see, e.g. Figure 3 ,

Now, referring again to Figure 2, for $i=1,2$ we let $\mathbf{e}_{i}$ denote the unit vector directed from $x_{3}$ to $x_{i}$ and we let $\mathbf{n}_{i}$ and $\mathbf{t}_{i}$ denote, respectively, the unit normal 


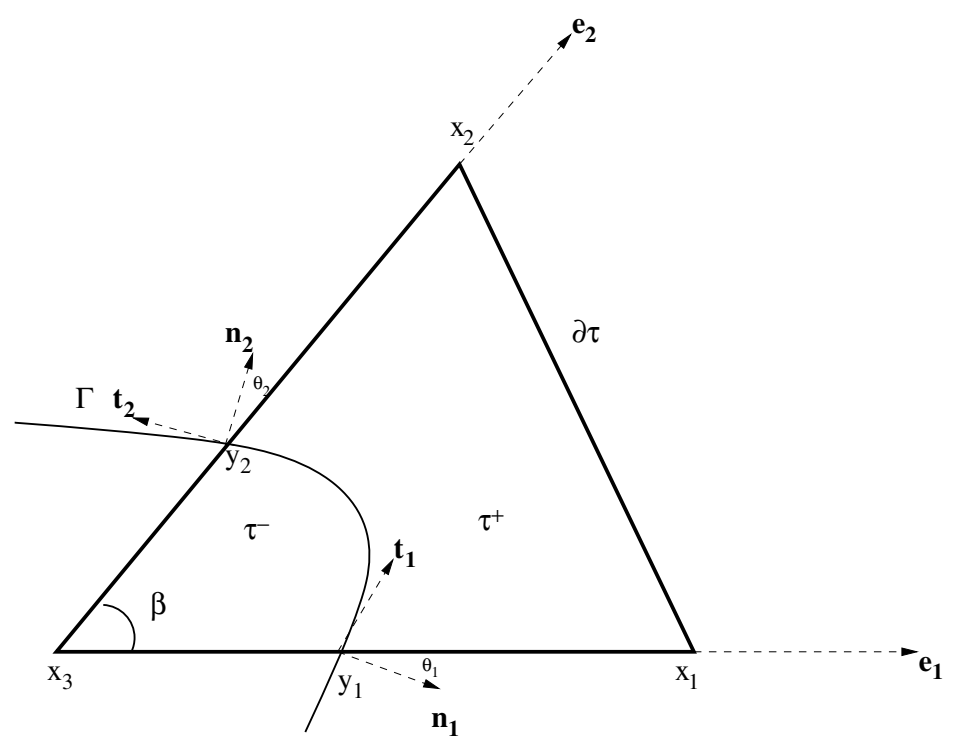

Figure 2. An interface cutting through an element: Here $\tau^{-}$denotes the part where the coefficient $\alpha$ is large.
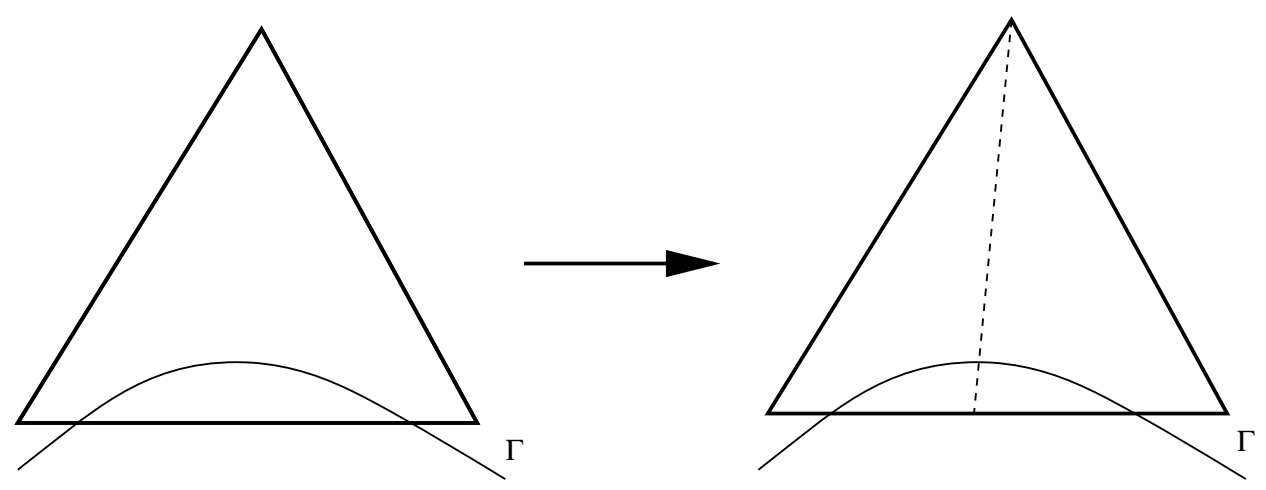

Figure 3. After a simple refinement, we can reduce the element to the case we consider.

and the unit tangent to $\Gamma$ at $y_{i}$. These are uniquely determined by requiring that $\mathbf{n}_{i}$ is directed outward from $\tau^{-}$and that $\mathbf{t}_{i}=R_{\pi / 2} \mathbf{n}_{i}$, where $R_{\phi}$ is the rotation matrix

$$
R_{\phi}=\left[\begin{array}{rr}
\cos \phi & -\sin \phi \\
\sin \phi & \cos \phi
\end{array}\right] \text {. }
$$

Note also that $\mathbf{e}_{i}=R_{\theta_{i}} \mathbf{n}_{i}$. In particular, $\mathbf{e}_{i}=\mathbf{n}_{i}$ if and only if $\theta_{i}=0$.

Later we shall use the easily derived relations:

$$
R_{\phi} \mathbf{n}_{i}=\cos \phi \mathbf{n}_{i}+\sin \phi \mathbf{t}_{i} \text { and } R_{\phi} \mathbf{t}_{i}=-\sin \phi \mathbf{n}_{i}+\cos \phi \mathbf{t}_{i}
$$

for $i=1,2$ and any $\phi$. From these it follows that $\mathbf{n}_{2}=R_{-\theta_{2}} \mathbf{e}_{2}=R_{-\theta_{2}+\beta} \mathbf{e}_{1}=$ $R_{\theta_{1}-\theta_{2}+\beta} \mathbf{n}_{1}$ and, similarly, $\mathbf{t}_{2}=R_{\theta_{1}-\theta_{2}+\beta} \mathbf{t}_{1}$. Combining these last two relations 
with (3.4) yields

$$
\mathbf{n}_{2}=\cos \left(\theta_{2}-\theta_{1}-\beta\right) \mathbf{n}_{1}-\sin \left(\theta_{2}-\theta_{1}-\beta\right) \mathbf{t}_{1}
$$

and

$$
\mathbf{t}_{2}=\sin \left(\theta_{2}-\theta_{1}-\beta\right) \mathbf{n}_{1}+\cos \left(\theta_{2}-\theta_{1}-\beta\right) \mathbf{t}_{1} .
$$

As explained above, we study here how the solution $u$ of (1.2) behaves on $\partial \tau$. This information will be used to construct suitable internal boundary conditions for multiscale basis functions in $\$ 3.2$

Throughout, we denote the restriction of $u$ on $\tau^{ \pm}$by $u^{ \pm}$. Also, for any unit vector $\mathbf{v}$ we let $D_{v} u$ denote the derivative of $u$ in the direction $\mathbf{v}$. The boundary conditions derived in the following section will be motivated by some relationships between the quantities $\left(D_{e_{i}} u^{ \pm}\right)\left(y_{i}\right)$, for $i=1,2$, where $u$ is the exact solution of (1.2). Our first relationship is the following:

Lemma 3.2. Let $u$ be the exact solution of (1.2) and define the vector $\varepsilon=$ $\left(\varepsilon_{1}, \varepsilon_{2}\right)^{T} \in \mathbb{R}^{2}$ by requiring

$$
r_{i}^{-}\left(D_{e_{i}} u^{-}\right)\left(y_{i}\right)+r_{i}^{+}\left(D_{e_{i}} u^{+}\right)\left(y_{i}\right)=u\left(x_{i}\right)-u\left(x_{3}\right)+\varepsilon_{i}, \quad i=1,2 .
$$

Then

$$
\left|\varepsilon_{i}\right| \lesssim h_{\tau}^{3 / 2}\left(\left\|D_{e_{i}}^{2} u^{-}\right\|_{L_{2}\left(e_{i} \cap \tau^{-}\right)}+\left\|D_{e_{i}}^{2} u^{+}\right\|_{L_{2}\left(e_{i} \cap \tau^{+}\right)}\right) \quad \text { for } \quad i=1,2 .
$$

Proof. This uses, in a straightforward way, the Taylor expansions at the point $y_{i}$ and the interface matching condition $u^{+}\left(y_{i}\right)=u^{-}\left(y_{i}\right)$, plus the fact that $u^{ \pm} \in$ $H^{2}\left(\tau^{ \pm}\right)$.

Now, if $u$ were known at the three node points $x_{i}, x_{2}, x_{3}$, then setting $\varepsilon_{i}=0$ in (3.7) would give us two equations for (approximations of) the four unknown quantities $\left(D_{e_{i}} u^{ \pm}\right)\left(y_{i}\right), i=1,2$.

To determine additional equations for these quantities, we use the interface jump conditions for $u$ at $y_{1}, y_{2}$ to obtain in a straightforward way:

Lemma 3.3. Let $u$ be the exact solution of (1.2). For $i=1,2$,

$$
\left[\begin{array}{c}
D_{e_{i}} u^{-}\left(y_{i}\right) \\
D_{e_{i}} u^{+}\left(y_{i}\right)
\end{array}\right]=A_{\widehat{\alpha}, \theta_{i}}\left[\begin{array}{c}
D_{n_{i}} u^{-}\left(y_{i}\right) \\
D_{t_{i}} u^{-}\left(y_{i}\right)
\end{array}\right],
$$

where

$$
A_{\widehat{\alpha}, \theta}=\left[\begin{array}{rr}
\cos \theta & \sin \theta \\
\widehat{\alpha} \cos \theta & \sin \theta
\end{array}\right] \text {. }
$$

Proof. The proof is obtained by simply combining the interface conditions:

$$
\left(D_{n_{i}} u^{+}\right)\left(y_{i}\right)=\widehat{\alpha}\left(D_{n_{i}} u^{-}\right)\left(y_{i}\right) \quad \text { and } \quad\left(D_{t_{i}} u^{+}\right)\left(y_{i}\right)=\left(D_{t_{i}} u^{-}\right)\left(y_{i}\right) \quad \text { for } i=1,2
$$

with (3.2).

We can use (3.9) to define a relation between $\left(D_{e_{i}} u^{ \pm}\right)\left(y_{i}\right), i=1,2$, provided we have a relation between $\left(D_{n_{1}} u^{-}\right)\left(y_{1}\right),\left(D_{t_{1}} u^{-}\right)\left(y_{1}\right),\left(D_{n_{2}} u^{-}\right)\left(y_{2}\right)$, and $\left(D_{t_{2}} u^{-}\right)\left(y_{2}\right)$. This is provided by the following lemma. 
Lemma 3.4. Let $u$ be the exact solution of (1.2):

$$
\left[\begin{array}{c}
D_{n_{2}} u^{-}\left(y_{2}\right) \\
D_{t_{2}} u^{-}\left(y_{2}\right)
\end{array}\right]=R_{\theta_{2}-\theta_{1}-\beta}\left[\begin{array}{c}
D_{n_{1}} u^{-}\left(y_{1}\right) \\
D_{t_{1}} u^{-}\left(y_{1}\right)
\end{array}\right]+\varepsilon^{\prime},
$$

where

$$
\left\|\varepsilon^{\prime}\right\|_{\infty} \lesssim h_{\tau}^{1 / 2}\left[\left\|D_{e_{2}} D_{n_{1}} u^{-}\right\|_{L_{2}\left(e_{2} \cap \tau^{-}\right)}^{2}+\left\|D_{e_{1}} D_{n_{1}} u^{-}\right\|_{L_{2}\left(e_{1} \cap \tau^{-}\right)}^{2}\right]^{1 / 2} .
$$

Proof. From equations (3.5) and (3.6) we have, for all $x \in \tau^{-}$,

$$
\left[\begin{array}{c}
D_{n_{2}} u^{-}(x) \\
D_{t_{2}} u^{-}(x)
\end{array}\right]=R_{\theta_{2}-\theta_{1}-\beta}\left[\begin{array}{c}
D_{n_{1}} u^{-}(x) \\
D_{t_{1}} u^{-}(x)
\end{array}\right] .
$$

Then, using Taylor expansions and the fact that $u^{-}$is $H^{2}$ on each $e_{i} \cap \tau^{-}$, we obtain the desired estimate.

Before we move on to the definition of the multiscale basis functions, in Corollary 3.5 we shall collect the results of Lemmas 3.2, 3.3 and 3.4 in a simpler form. To do this, introduce the $6 \times 6$ matrix

$$
M_{\widehat{\alpha}, \theta_{1}, \theta_{2}, \beta}:=\left[\begin{array}{rrl}
I & 0 & -A_{\widehat{\alpha}, \theta_{1}} \\
0 & I & -A_{\widehat{\alpha}, \theta_{2}} R_{\theta_{2}-\theta_{1}-\beta} \\
\mathcal{R}_{1} & \mathcal{R}_{2} & 0
\end{array}\right],
$$

where

$$
\mathcal{R}_{1}=\left[\begin{array}{rr}
r_{1}^{-} & r_{1}^{+} \\
0 & 0
\end{array}\right] \quad \text { and } \quad \mathcal{R}_{2}=\left[\begin{array}{cc}
0 & 0 \\
r_{2}^{-} & r_{2}^{+}
\end{array}\right]
$$

Also, for each $v \in H_{0}^{1}(\Omega)$ with suitably well-defined point values at $y_{i}, i=1,2$, we define the vectors $\mathbf{c}(v) \mathbf{d}(v) \in \mathbb{R}^{6}$ by

$$
\mathbf{c}(v)=\left[0,0,0,0, v\left(x_{1}\right)-v\left(x_{3}\right), v\left(x_{2}\right)-v\left(x_{3}\right)\right]^{T}
$$

and

$$
\begin{aligned}
\mathbf{d}(v):= & {\left[\left(D_{e_{1}} v^{-}\right)\left(y_{1}\right),\left(D_{e_{1}} v^{+}\right)\left(y_{1}\right),\left(D_{e_{2}} v^{-}\right)\left(y_{2}\right),\left(D_{e_{2}} v^{+}\right)\left(y_{2}\right),\right.} \\
& \left.\left(D_{n_{1}} v^{-}\right)\left(y_{1}\right),\left(D_{t_{1}} v^{-}\right)\left(y_{1}\right)\right]^{T} .
\end{aligned}
$$

Note that $\mathbf{c}(v)$ and $\mathbf{d}(v)$ depend linearly on $v$. Then we have

Corollary 3.5. If $u$ is the exact solution of the problem (1.2), then for each element $\tau$ which intersects the interface as in Assumption 3.1, and with the notation defined there, we have

$$
M_{\widehat{\alpha}, \theta_{1}, \theta_{2}, \beta} \mathbf{d}(u)=\mathbf{c}(u)+\boldsymbol{\delta},
$$

where $\boldsymbol{\delta} \in \mathbb{R}^{6}$ is defined by

$$
\boldsymbol{\delta}=\left[\begin{array}{c}
\mathbf{0} \\
A_{\widehat{\alpha}, \theta_{2}} \varepsilon^{\prime} \\
\varepsilon
\end{array}\right],
$$

and $\varepsilon, \varepsilon^{\prime}$ are as defined in Lemmas 3.2 and 3.4 .

Proof. This is obtained by writing: (i) Lemma 3.3 for $i=1$; (ii) Lemma 3.3 for $i=2$ combined with Lemma 3.4; (iii) Lemma 3.2 . 
3.2. Novel interior boundary condition and boundary error. We now use the relations derived in the previous subsection to derive suitable boundary conditions for multiscale basis functions.

For any element $\tau \in \mathcal{T}_{h}$, let $x_{p}, p=1,2,3$, denote its nodes. The multiscale basis functions $\Phi_{p}^{\mathrm{MS}}$ are found as solutions to the subgrid problems (2.1) on $\tau$, subject to Dirichlet boundary data $\phi_{p, \tau}$ on $\partial \tau$ which has to be specified, subject to the nodal condition

$$
\phi_{p, \tau}\left(x_{q}\right)=\delta_{p, q}, \quad p, q \in\{1,2,3\}
$$

(see (2.2) ). If the interface $\Gamma$ does not intersect $\tau$, then we choose $\phi_{p, \tau}$ on $\partial \tau$ to be the linear interpolant of (3.18) on each edge of $\tau$, and then the solution of (2.1) is also linear on $\tau$. Otherwise (under Assumption 3.1), our construction for $\phi_{p, \tau}$ (described below) will be continuous on $\partial \tau$, linear on each of the intersected edge segments $\left\{\overline{x_{3} y_{i}}, \quad \overline{y_{i} x_{i}}, \quad\right.$ for $\left.\quad i=1,2\right\}$ and linear on the third edge $\overline{x_{1} x_{2}}$. Because of (3.18), it remains to specify the gradient of $\phi_{p, \tau}$ on each of the two pieces of the intersected edges. These gradients are computed by Algorithm 3.7 below. This requires solving two $6 \times 6$ linear systems with the same coefficient matrices. Before we give the algorithm we first establish the solvability of these systems and obtain bounds on their solution which will be needed later.

Theorem 3.6. Under Assumption [3.1, suppose $\phi:=\theta_{2}-\theta_{1}-\beta \neq 0$ and introduce the $2 \times 2$ matrix

$$
D:=\mathcal{R}_{1} A_{\widehat{\alpha}, \theta_{1}}+\mathcal{R}_{2} A_{\widehat{\alpha}, \theta_{2}} R_{\phi} .
$$

Then, for all $\widehat{\alpha}$ sufficiently large, $D$ is non-singular, and $M_{\widehat{\alpha}, \theta_{1}, \theta_{2}, \beta}$ is non-singular with

$$
\left(M_{\widehat{\alpha}, \theta_{1}, \theta_{2}, \beta}\right)^{-1}=\left[\begin{array}{ccc}
I & 0 & A_{\widehat{\alpha}, \theta_{1}} \\
0 & I & A_{\widehat{\alpha}, \theta_{2}} R_{\phi} \\
0 & 0 & I
\end{array}\right]\left[\begin{array}{ccc}
I & 0 & 0 \\
0 & I & 0 \\
-D^{-1} \mathcal{R}_{1} & -D^{-1} \mathcal{R}_{2} & D^{-1}
\end{array}\right]
$$

Moreover,

$$
\left\|D^{-1}\right\|_{\infty} \lesssim \widehat{\alpha}^{-1} h_{\tau}^{-1}(\sin \phi)^{-1}
$$

Proof. A tedious but elementary calculation shows that with

$$
E:=\left[\begin{array}{cl}
r_{1}^{+} \cos \theta_{1} & 0 \\
r_{2}^{+} \cos \theta_{2} \cos \phi & -r_{2}^{+} \cos \theta_{2} \sin \phi
\end{array}\right]
$$

we have $\left\|\widehat{\alpha}^{-1} D-E\right\|_{\infty} \leq C \widehat{\alpha}^{-1} h_{\tau}$, with the constant $C$ independent of $\theta_{1}, \theta_{2}$, $\phi, \beta$ and $h_{\tau}$. Since $E$ is non-singular, standard matrix perturbation theory shows that, for large enough $\widehat{\alpha}$,

$$
\left\|\widehat{\alpha} D^{-1}\right\|_{\infty}=\left\|\left(\widehat{\alpha}^{-1} D\right)^{-1}\right\|_{\infty} \leq C^{\prime}\left\|E^{-1}\right\|_{\infty}
$$

with $C^{\prime}$ also independent of the above parameters. In fact,

$$
E^{-1}=\left[\begin{array}{cc}
\left(r_{1}^{+} \cos \theta_{1}\right)^{-1} & 0 \\
-\left(r_{1}^{+} \cos \theta_{1} \sin \phi\right)^{-1} \cos \phi & -\left(r_{2}^{+} \cos \theta_{2} \sin \phi\right)^{-1}
\end{array}\right],
$$

and so (3.20) follows directly, recalling Assumption 3.1. Because $D^{-1}$ exists, the formula for $M^{-1}$ is verified by simple matrix manipulation. 
This now leads us to Algorithm 3.7 for computing the boundary data $\phi_{p, \tau}$ for the multiscale basis functions $\Phi_{p}^{\mathrm{MS}}$ on $\tau$.

Algorithm 3.7. For $p=1,2,3$,

(1) Solve the linear system:

$$
M_{\widehat{\alpha}, \theta_{1}, \theta_{2}, \beta} \mathbf{d}_{p}=\mathbf{c}\left(\phi_{p, \tau}\right) .
$$

(2) Then set

$$
\begin{cases}\left.\left(D_{e_{1}} \phi_{p, \tau}\right)\right|_{\overline{x_{3} y_{1}}}=\left(\mathbf{d}_{p}\right)_{1}, \quad & \left.\left(D_{e_{1}} \phi_{p, \tau}\right)\right|_{\overline{y_{1} x_{1}}}=\left(\mathbf{d}_{p}\right)_{2} \\ \left.\left(D_{e_{2}} \phi_{p, \tau}\right)\right|_{\overline{x_{3} y_{2}}}=\left(\mathbf{d}_{p}\right)_{3}, & \left.\left(D_{e_{2}} \phi_{p, \tau}\right)\right|_{\overline{y_{2} x_{2}}}=\left(\mathbf{d}_{p}\right)_{4} .\end{cases}
$$

Remark 3.8. (i) The right-hand side $\mathbf{c}\left(\phi_{p, \tau}\right)$ in system (3.21) is determined by (3.15) and (3.18). It is easy to see that $\mathbf{c}\left(\phi_{1, \tau}\right)+\mathbf{c}\left(\phi_{2, \tau}\right)+\mathbf{c}\left(\phi_{3, \tau}\right)=\mathbf{0}$, so $\mathbf{d}_{1}+\mathbf{d}_{2}+\mathbf{d}_{3}=\mathbf{0}$ and only two of the three systems (3.21) has to be solved. Moreover, since the function $\phi_{1, \tau}+\phi_{2, \tau}+\phi_{3, \tau}$ has value 1 at each node of $\tau$ and zero derivative along each $\overline{x_{3} y i}, \overline{y_{i} x_{i}}, i=1,2$, and along $\overline{x_{1} x_{2}}$, it thus satisfies the requirements of (2.2) and (2.3).

(ii) Since $\phi_{p, \tau}$ is defined to be linear on each $e_{i} \cap \tau^{-}$and $e_{i} \cap \tau^{+}$and to satisfy the nodal condition (3.18), the continuity of $\phi_{p, \tau}$ at each intersection point $y_{i}$ is guaranteed by the last two equations in (3.21).

(iii) If $\theta_{i}=0$ (i.e. the interface intersects edge $e_{i}$ orthogonally), then the boundary condition computed by Algorithm 3.7 coincides with the "oscillatory boundary condition" proposed in [19. More precisely, if $\theta_{1}=0$, it is easy to see that the first two equations and last two equations of (3.21) imply

$\left(\mathbf{d}_{p}\right)_{2}=\widehat{\alpha}\left(\mathbf{d}_{p}\right)_{1} \quad$ and $\quad \mathrm{r}_{1}^{-}\left(\mathbf{d}_{p}\right)_{1}+r_{1}^{+}\left(\mathbf{d}_{p}\right)_{2}=\phi_{p, \tau}\left(x_{1}\right)-\phi_{p, \tau}\left(x_{3}\right)$,

and hence

$$
\left(\mathbf{d}_{p}\right)_{1}=\frac{\phi_{p, \tau}\left(x_{1}\right)-\phi_{p, \tau}\left(x_{3}\right)}{r_{1}^{-}+\widehat{\alpha} r_{1}^{+}}, \quad\left(\mathbf{d}_{p}\right)_{2}=\widehat{\alpha} \frac{\phi_{p, \tau}\left(x_{1}\right)-\phi_{p, \tau}\left(x_{3}\right)}{r_{1}^{-}+\widehat{\alpha} r_{1}^{+}} .
$$

Thus $\phi_{p, \tau}$ is the solution of the reduced elliptic differential equation

$$
-\left(\alpha \phi_{p, \tau}^{\prime}\right)^{\prime}=0
$$

on $\overline{x_{3} x_{1}}$, which is exactly how the "oscillatory" boundary condition is constructed.

(iv) When $\theta_{i} \neq 0$ for $i=1,2$ the boundary condition on each $e_{i}$ depends on both $\theta_{1}$ and $\theta_{2}$. In particular, if $p=1$ (respectively 2), the function $\phi_{p, \tau}$ does not necessarily vanish on the edge $e_{2}$ (respectively $e_{1}$ ).

(v) Algorithm 3.7 determines $\phi_{p, \tau}$ and hence $\Phi_{p}^{\mathrm{MS}}$ on each $\tau$ individually and does not guarantee that $\Phi_{p}^{\mathrm{MS}}$ will be continuous across element edges, so that approximation in $\operatorname{span}\left\{\Phi_{p}^{\mathrm{MS}}: p \in \mathscr{I}_{h}(\Omega)\right\}$ may not be conforming. We resolve this issue later by averaging across element edges (see 3.4).

In the next theorem we show that the nodal interpolant $\mathcal{I}_{h}^{\mathrm{MS}} u=\sum_{p} u(p) \phi_{p, \tau}$ is a good approximation to $u$ along the boundary of the element $\tau$. Recall the notation $E_{h}^{\mathrm{MS}}:=u-\mathcal{I}_{h}^{\mathrm{MS}} u$. 
Theorem 3.9. Let $u$ be the exact solution of (1.2). Consider an element $\tau$ which intersects the interface as in Assumption 3.1. Suppose also that $\phi:=\theta_{2}-\theta_{1}-\beta \neq$ 0 . Then we have, for $m=0,1$,

$$
\begin{aligned}
\max _{i=1,2} & \left\{\widehat{\alpha} h_{\tau}^{m}\left\|D_{e_{i}}^{m} E_{h}^{\mathrm{MS}}\right\|_{L_{\infty}\left(e_{i} \cap \tau^{-}\right)}, h_{\tau}^{m}\left\|D_{e_{i}}^{m} E_{h}^{\mathrm{MS}}\right\|_{L_{\infty}\left(e_{i} \cap \tau^{+}\right)}\right\} \\
& \lesssim h_{\tau}^{3 / 2} \max _{\substack{i=1,2 \\
|k|=1}}\left[\widehat{\alpha}^{2}\left\|D^{k} D_{e_{i}} u\right\|_{L_{2}\left(e_{i} \cap \tau^{-}\right)}^{2}+\left\|D^{k} D_{e_{i}} u\right\|_{L_{2}\left(e_{i} \cap \tau^{+}\right)}^{2}\right]^{1 / 2} .
\end{aligned}
$$

The hidden constant may blow up if $\phi \rightarrow 0$. (See Remark 3.10 below.)

Proof. We give the proof on the assumption that $\tau^{ \pm}$are as depicted in Figure2(i.e. $\alpha$ is large in the region containing $x_{3}$ and small in the region containing $x_{1}, x_{2}$ ). Making use of (3.21) and the fact that $\mathbf{c}(u)$ depends only on the nodal values of $u$, we have

$$
M_{\widehat{\alpha}, \theta_{1}, \theta_{2}, \beta}\left(\sum_{p=1}^{3} u\left(x_{p}\right) \mathbf{d}_{p}\right)=\mathbf{c}\left(\mathcal{I}_{h}^{\mathrm{MS}} u\right)=\mathbf{c}(u) .
$$

Combining this with Corollary 3.5 we obtain

$$
M_{\widehat{\alpha}, \theta_{1}, \theta_{2}, \beta}\left(\mathbf{d}(u)-\sum_{p=1}^{3} u\left(x_{p}\right) \mathbf{d}_{p}\right)=\boldsymbol{\delta} .
$$

Hence, using (3.19) and (3.17), we obtain

$$
\left(\mathbf{d}(u)-\sum_{p=1}^{3} u\left(x_{p}\right) \mathbf{d}_{p}\right)=\left[\begin{array}{ccc}
I & 0 & A_{\widehat{\alpha}, \theta_{1}} \\
0 & I & A_{\widehat{\alpha}, \theta_{2}} R_{\phi} \\
0 & 0 & I
\end{array}\right]\left[\begin{array}{c}
\mathbf{0} \\
A_{\widehat{\alpha}, \theta_{2}} \varepsilon^{\prime} \\
D^{-1}\left(\varepsilon-\mathcal{R}_{2} A_{\widehat{\alpha}, \theta_{2}} \varepsilon^{\prime}\right)
\end{array}\right] .
$$

Now by (3.16) and (3.22), we see that the first four entries of the left-hand side of (3.25) are

$$
D_{e_{1}}\left(u-\mathcal{I}_{h}^{\mathrm{MS}} u\right)^{-}\left(y_{1}\right), D_{e_{1}}\left(u-\mathcal{I}_{h}^{\mathrm{MS}} u\right)^{+}\left(y_{1}\right), D_{e_{2}}\left(u-\mathcal{I}_{h}^{\mathrm{MS}} u\right)^{-}\left(y_{2}\right),
$$

and

$$
D_{e_{2}}\left(u-\mathcal{I}_{h}^{\mathrm{MS}} u\right)^{+}\left(y_{2}\right)
$$

Examining the right-hand side of (3.25), we see that the first two entries are

$$
A_{\widehat{\alpha}, \theta_{1}} D^{-1}\left(\varepsilon-\mathcal{R}_{2} A_{\widehat{\alpha}, \theta_{2}} \varepsilon^{\prime}\right) .
$$

Now, recalling Lemmas $3.2,3.4$ and (3.20) we obtain

$$
\begin{aligned}
& \left\|D^{-1}\left(\varepsilon-\mathcal{R}_{2} A_{\widehat{\alpha}, \theta_{2}} \varepsilon^{\prime}\right)\right\|_{\infty} \\
& \quad \lesssim h_{\tau}^{1 / 2}(\sin \phi)^{-1} \max _{\substack{i=1,2 \\
|k|=1}}\left\{\left\|D^{k} D_{e_{i}} u\right\|_{L_{2}\left(e_{i} \cap \tau^{-}\right)}^{2}+\widehat{\alpha}^{-2}\left\|D^{k} D_{e_{i}} u\right\|_{L_{2}\left(e_{i} \cap \tau^{+}\right)}^{2}\right\}^{1 / 2} .
\end{aligned}
$$

Hence

$$
\begin{aligned}
& \max \left\{\widehat{\alpha}\left|D_{e_{1}}\left(u-\mathcal{I}_{h}^{\mathrm{MS}} u\right)^{-}\left(y_{1}\right)\right|,\left|D_{e_{1}}\left(u-\mathcal{I}_{h}^{\mathrm{MS}} u\right)^{+}\left(y_{1}\right)\right|\right\} \\
& \quad \lesssim h_{\tau}^{1 / 2} \max _{\substack{i=1,2 \\
|k|=1}}\left[\widehat{\alpha}^{2}\left\|D^{k} D_{e_{i}} u\right\|_{L_{2}\left(e_{i} \cap \tau^{-}\right)}^{2}+\left\|D^{k} D_{e_{i}} u\right\|_{L_{2}\left(e_{i} \cap \tau^{+}\right)}^{2}\right]^{1 / 2}
\end{aligned}
$$


Similarly, the third and fourth components of (3.25) yield the same estimate for $\max \left\{\widehat{\alpha}\left|D_{e_{2}}\left(u-\mathcal{I}_{h}^{\mathrm{MS}} u\right)^{-}\left(y_{2}\right)\right|,\left|D_{e_{2}}\left(u-\mathcal{I}_{h}^{\mathrm{MS}} u\right)^{+}\left(y_{2}\right)\right|\right\}$.

The estimates (3.24) for $m=1$ then readily follow. For example, since $\mathcal{I}_{h}^{\mathrm{MS}} u$ is linear on $e_{i} \cap \tau^{-}$, we have, for $x \in e_{i} \cap \tau^{-}$,

$$
\begin{aligned}
\widehat{\alpha} \mid D_{e_{i}} & \left(u-\mathcal{I}_{h}^{\mathrm{MS}} u\right)(x) \mid \\
& \leq \widehat{\alpha}\left|\left(D_{e_{i}} u\right)(x)-\left(D_{e_{i}} u^{-}\right)\left(y_{i}\right)\right|+\widehat{\alpha}\left|D_{e_{i}}\left(u-\mathcal{I}_{h}^{\mathrm{MS}} u\right)^{-}\left(y_{i}\right)\right| \\
& \lesssim h_{\tau}^{1 / 2} \max _{\substack{i=1,2 \\
|k|=1}}\left[\widehat{\alpha}^{2}\left\|D^{k} D_{e_{i}} u\right\|_{L_{2}\left(e_{i} \cap \tau^{-}\right)}^{2}+\left\|D^{k} D_{e_{i}} u\right\|_{L_{2}\left(e_{i} \cap \tau^{+}\right)}^{2}\right]^{1 / 2} .
\end{aligned}
$$

To obtain the estimate for $m=0$, recall that $u-\mathcal{I}_{h}^{\mathrm{MS}} u$ vanishes at the nodes, so we can write, for $x \in e_{1} \cap \tau^{-}$,

$$
\left(u-\mathcal{I}_{h}^{\mathrm{MS}} u\right)(x)=\int_{x_{3}}^{x} D_{e_{1}}\left(u-\mathcal{I}_{h}^{\mathrm{MS}} u\right)(t) d t,
$$

and the required estimates for $\left\|u-\mathcal{I}_{h}^{\mathrm{MS}} u\right\|_{L_{\infty}\left(e_{1} \cap \tau^{-}\right)}$follow directly. The remainder of the estimates (3.24) for $m=0$ are similar.

Remark 3.10. The critical case $\phi=\theta_{2}-\theta_{1}-\beta=0$ in Theorem 3.9 occurs when the unit outward normals $\mathbf{n}_{1}$ and $\mathbf{n}_{2}$ to $\Gamma$ at the two intersection points $\mathbf{y}_{1}, \mathbf{y}_{2}$ coincide. In this case, if the interface $\Gamma$ is not a straight line, then $\tau$ may be subdivided into two sub-elements, in each of which $\phi$ no longer vanishes, and Algorithm 3.7 applies to each of these sub-elements.

However, if $\Gamma \cap \tau$ is a straight line, no such refinement will succeed. Instead (referring to the geometry in Figure 2), we may simply subdivide the quadrilateral $\tau^{+}$into two triangles and combine this with $\tau^{-}$to yield a new mesh which locally resolves $\Gamma$, and then we can discretise using standard linear basis functions on each of these three elements.

An alternative approach is suggested by the "Immersed Finite Element (IFE) method" of $\mathrm{Li}, \mathrm{Lin}$ and $\mathrm{Wu} 28$, where in any case the interface segment $\tau \cap \Gamma$ is approximated by a straight line and where a special finite element basis $\Psi_{p, \tau}: p=$ $1,2,3$ is constructed on $\tau$, which is required to be affine on each of $\tau^{-}$and $\tau^{+}$and to satisfy the six conditions (with the same geometry as in Assumption 3.1)

$$
\begin{aligned}
\Psi_{p, \tau}\left(x_{q}\right) & =\delta_{p, q}, \quad q=1,2,3, \\
\Psi_{p, \tau}^{-}\left(y_{i}\right) & =\Psi_{p, \tau}^{+}\left(y_{i}\right), \quad i=1,2, \\
\text { and } D_{n} \Psi_{p, \tau}^{-} & =\widehat{\alpha} D_{n} \Psi_{p, \tau}^{+} .
\end{aligned}
$$

Here $\mathbf{n}$ denotes the (constant) normal direction to the straight line $\Gamma \cap \tau$ pointing from $\tau^{-}$to $\tau^{+}$and $\mathbf{t}$ the corresponding tangential direction (as in Figure 22). Note that in (3.30), the quantities on each side of the equation are constant since the $\Psi_{p, \tau}^{ \pm}$are assumed affine.

The following lemma shows that the immersed finite element algorithm defines a solution to (3.21) even when $\Gamma \cap \tau$ is a straight line (so that $\phi=0$ ). However, the error estimates of Theorem 3.9 are no longer true in general for the IFE approach, as the following example shows.

Example 3.11. Consider an element $\tau$ with vertices $(0,0),(0, h),(h, h)$ and where the interface is the segment connected by $(0, h / 2),(h / 2, h / 2)$. If we consider 
$\alpha=\widehat{\alpha}$ on $\tau^{-}$(the left part) and $\alpha=1$ on $\tau^{+}$(the right part), then the IFE nodal basis function with value 1 at $(h, 0)$ is given by

$$
\frac{2 x}{(\widehat{\alpha}+1) h}-\frac{y}{h} \quad \text { on } \quad \tau^{-} \quad \text { and } \quad \frac{2 \widehat{\alpha}(x-h)}{(\widehat{\alpha}+1) h}-\frac{y-h}{h} \text { on } \tau^{+} .
$$

The directional derivative along the edge from $(0,0),(h, h)$ in $\tau^{-}$is $\frac{\sqrt{2}}{(\widehat{\alpha}+1) h}-\frac{1}{\sqrt{2} h}$ of $\mathcal{O}(1 / h)$ but not $\mathcal{O}(1 /(\widehat{\alpha} h))$. Hence the estimate in Theorem 3.9 can not be true.

Lemma 3.12. Suppose $\Gamma \cap \tau$ is a straight line segment and suppose $\Psi_{p, \tau}$ is defined via (3.28), (3.29) and (3.30). Then the vector $\mathbf{d}\left(\Psi_{p, \tau}\right)$ (defined as in (3.16)) provides a solution to system (3.21).

Proof. Using the fact that $\Psi_{p, \tau}$ is affine on each of $\tau^{ \pm}$and also using (3.29), we have, for $i=1,2$,

$$
\begin{aligned}
& \left(\nabla \Psi_{p, \tau}^{-}\right)^{T}\left(y_{i}-x_{3}\right)+\left(\nabla \Psi_{p, \tau}^{+}\right)^{T}\left(x_{i}-y_{i}\right) \\
& \quad=\Psi_{p, \tau}^{-}\left(y_{i}\right)-\Psi_{p, \tau}\left(x_{3}\right)+\Psi_{p, \tau}\left(x_{i}\right)-\Psi_{p, \tau}^{+}\left(y_{i}\right) \\
& \quad=\Psi_{p, \tau}\left(x_{i}\right)-\Psi_{p, \tau}\left(x_{3}\right) .
\end{aligned}
$$

Since $r_{i}^{-} \mathbf{e}_{i}=\left(y_{i}-x_{3}\right)$ and $r_{i}^{+} \mathbf{e}_{i}=\left(x_{i}-y_{i}\right)$, and since the gradients $\nabla \Psi_{p, \tau}^{ \pm}$are constant, it follows that

$$
r_{i}^{-} D_{e_{i}} \Psi_{p, \tau}^{-}\left(y_{i}\right)+r_{i}^{+} D_{e_{i}} \Psi_{p, \tau}^{+}\left(y_{1}\right)=\Psi_{p, \tau}\left(x_{i}\right)-\Psi_{p, \tau}\left(x_{3}\right) \quad \text { for } \quad i=1,2,
$$

and so the last two equations of (3.21) are satisfied.

By a similar argument,

$$
\left(\nabla \Psi_{p, \tau}^{-}\right)^{T}\left(y_{1}-y_{2}\right)=\Psi_{p, \tau}^{-}\left(y_{1}\right)-\Psi_{p, \tau}^{-}\left(y_{2}\right)=\Psi_{p, \tau}^{+}\left(y_{1}\right)-\Psi_{p, \tau}^{+}\left(y_{2}\right)=\left(\nabla \Psi_{p, \tau}^{+}\right)^{T}\left(y_{1}-y_{2}\right),
$$

and since $y_{1}-y_{2}$ is in the direction of $t$ (the tangent direction along $\Gamma$ ), this implies

$$
D_{t} \Psi_{p, \tau}^{+}=D_{t} \Psi_{p, \tau}^{-} \quad \text { on } \quad \Gamma \cap \tau .
$$

Combining this with (3.2) and (3.30), we have

$$
\begin{aligned}
& D_{e_{1}} \Psi_{p, \tau}^{-}=\cos \theta_{1} D_{n} \Psi_{p, \tau}^{-}+\sin \theta_{1} D_{t} \Psi_{p, \tau}^{-}, \\
& D_{e_{1}} \Psi_{p, \tau}^{+}=\widehat{\alpha} \cos \theta_{1} D_{n} \Psi_{p, \tau}^{-}+\sin \theta_{1} D_{t} \Psi_{p, \tau}^{-},
\end{aligned}
$$

and thus the first two equations in (3.21) are satisfied. The verification of the third and fourth equations in (3.21) is entirely analogous.

Remark 3.13. The previous lemma shows that the system (3.21) is consistent when $\Gamma \cap \tau$ is a straight line, which is a particular case of $\phi=0$. Under the general assumption only that $\phi=0$ and by examining the proof of Theorem 3.6. we see that in this case $D=\mathcal{R}_{1} A_{\widehat{\alpha}, \theta_{1}}+\mathcal{R}_{2} A_{\widehat{\alpha}, \theta_{2}}$. The (non)singularity of this for general choices of $\widehat{\alpha}$ and for $\theta_{i}, r_{i}^{-}, r_{i}^{+}, i=1,2$, has not yet been analysed.

3.3. Interior error. The main result in this section is Theorem 3.16, which gives an $\alpha$-explicit estimate for the error $\left|u-u_{h}^{\mathrm{MS}}\right|_{H^{1}(\tau), \alpha}$, in the case where the interface may cut through $\tau$. This is obtained by an application of Theorem 2.2 and thus requires that we first show that $E_{h}^{\mathrm{MS}}=u-\mathcal{I}_{h}^{\mathrm{MS}} u$ can be extended from the boundary to the interior of $\tau$ in a suitably robust way. This extension is proved in Lemma 3.15, which requires a further technical assumption on the geometry of $\Gamma \cap \tau$. 
Assumption 3.14. We impose Assumption 3.1, and we further assume that when $\Gamma$ intersects any element $\tau, \Gamma \cap \tau$ is star-shaped about $x_{3}$. That is, introducing polar coordinates with origin $x_{3}$ and polar angle $\theta$ measured counterclockwise from $e_{1}$, we assume that each $(x, y) \in \Gamma \cap \tau$ can be written as $(x, y)=(r(\theta) \cos \theta, r(\theta) \sin \theta)$, for $\theta \in[0, \beta]$. Also writing the edge $\overline{x_{1} x_{2}}$ as $(x, y)=\left(r^{*}(\theta) \cos \theta, r^{*}(\theta) \sin \theta\right)$ for a suitable function $r^{*}$, we assume that there exist constants $C>0$ and $1>C^{*}>0$ such that

$$
\left|r^{\prime}(\theta)\right| \leq C r(\theta) \text { and } r(\theta) \leq C^{*} r^{*}(\theta) \text { for all } \theta \in[0, \beta] .
$$

Note that under this assumption, we can integrate the left-hand side of (3.31) to obtain $|\log (r(\theta) / r(0))| \leq C \beta$, and since $r(0)=r_{1}^{-}$, we can combine this with (3.1) to obtain

$$
r(\theta) \sim h_{\tau} \quad \text { for all } \theta \in[0, \beta] .
$$

Now letting $s$ denote arclength along $\Gamma \cap \tau$, it is easily seen that

$$
d s=\sqrt{(r(\theta))^{2}+\left(r^{\prime}(\theta)\right)^{2}} d \theta \sim h_{\tau} d \theta .
$$

Moreover, since (3.31) implies $r^{*}(\theta) \sim h_{\tau}$, we also have

$$
\left|\tau^{+}\right|=\int_{0}^{\beta} \int_{r(\theta)}^{r_{1}(\theta)} r d r d \theta=\frac{1}{2} \int_{0}^{\beta}\left[\left(r^{*}\right)^{2}(\theta)-r^{2}(\theta)\right] d \theta \sim h_{\tau}^{2} .
$$

A similar but simpler argument shows that $\left|\tau^{-}\right| \sim h_{\tau}^{2}$. Collecting these relations, we have

$$
|\Gamma \cap \tau| \sim h_{\tau}, \quad\left|\tau^{ \pm}\right| \sim h_{\tau}^{2} .
$$

These are needed in the proof of the following result.

Lemma 3.15. Under Assumption 3.14 there exists $\widetilde{E}_{h}^{\mathrm{MS}} \in H^{1}(\tau)$ with $\widetilde{E}_{h}^{\mathrm{MS}}=$ $E_{h}^{\mathrm{MS}}$ on $\partial \tau$ and satisfying

$$
\left|\widetilde{E}_{h}^{\mathrm{MS}}\right|_{H^{1}(\tau), \alpha}^{2} \lesssim h_{\tau}^{2}\left(\widehat{\alpha} \max _{i=1,2,3}\left\|D_{e_{i}} E_{h}^{\mathrm{MS}}\right\|_{L_{\infty}\left(e_{i} \cap \tau^{-}\right)}^{2}+\max _{i=1,2,3}\left\|D_{e_{i}} E_{h}^{\mathrm{MS}}\right\|_{L_{\infty}\left(e_{i} \cap \tau^{+}\right)}^{2}\right) .
$$

Proof. For notational convenience in the proof we make the following abbreviations: $E=E_{h}^{\mathrm{MS}}, \widetilde{E}=\widetilde{E}_{h}^{\mathrm{MS}}$. We assume the geometric situation as in Figure 2, so that $\tau^{-}$(the region where $\alpha$ is high) contains the node $x_{3}$. The case where $\tau^{-}$contains two nodes is entirely analogous.

Using Assumption 3.14, we can parametrise $\tau^{-}$by introducing local coordinates $(t, \theta)$ such that

$$
x=\operatorname{tr}(\theta) \cos \theta, \quad y=\operatorname{tr}(\theta) \sin \theta, \quad t \in[0,1], \quad \theta \in[0, \beta] .
$$

Then we define $\widetilde{E}$ on $\tau^{-}$explicitly by

$\widetilde{E}(t, \theta)=\left(\frac{\theta}{\beta}\right) E\left(x_{3}+t r_{2}^{-} \mathbf{e}_{2}\right)+\left(1-\frac{\theta}{\beta}\right) E\left(x_{3}+t r_{1}^{-} \mathbf{e}_{1}\right), \quad t \in[0,1], \quad \theta \in[0, \beta]$. 
Clearly $\widetilde{E}$ coincides with $E$ on $e_{i} \cap \tau^{-}$for each $i=1,2$ and, moreover,

$$
\begin{aligned}
\frac{\partial \widetilde{E}}{\partial x}(t, \theta)=( & \left(\frac{\theta}{\beta}\right) r_{2}^{-}\left(D_{e_{2}} E\right)\left(x_{3}+t r_{2}^{-} \mathbf{e}_{2}\right) \\
& \left.+\left(1-\frac{\theta}{\beta}\right) r_{1}^{-}\left(D_{e_{1}} E\right)\left(x_{3}+t r_{1}^{-} \mathbf{e}_{1}\right)\right) \frac{\partial t}{\partial x} \\
+ & \frac{1}{\beta}\left(E\left(x_{3}+t r_{2}^{-} \mathbf{e}_{2}\right)-E\left(x_{3}+t r_{1}^{-} \mathbf{e}_{1}\right)\right) \frac{\partial \theta}{\partial x}
\end{aligned}
$$

with an analogous formula for $\partial \widetilde{E} / \partial y$. Defining the Jacobian

$$
J:=\left[\begin{array}{cc}
\frac{\partial x}{\partial t} & \frac{\partial x}{\partial \theta} \\
\frac{\partial y}{\partial t} & \frac{\partial y}{\partial \theta}
\end{array}\right], \quad \text { we have } \operatorname{det}(J)=t r^{2}(\theta),
$$

and (abbreviating $r(\theta)$ by $r$ ) the partial derivatives of $\theta$ and $t$ may then be computed by

$$
\left[\begin{array}{ll}
\frac{\partial t}{\partial x} & \frac{\partial t}{\partial y} \\
\frac{\partial \theta}{\partial x} & \frac{\partial \theta}{\partial y}
\end{array}\right]=J^{-1}=\frac{1}{t r^{2}}\left[\begin{array}{cc}
t\left(r \cos \theta+r^{\prime} \sin \theta\right) & t\left(r \sin \theta-r^{\prime} \cos \theta\right) \\
-r \sin \theta & r \cos \theta
\end{array}\right]
$$

Making use of (3.40) and then Assumption 3.14 (which includes Assumption 3.1), the first term on the right-hand side of (3.38) may be estimated by

$$
\frac{h_{\tau}}{r(\theta)}\left|\cos \theta+\frac{r^{\prime}(\theta)}{r(\theta)} \sin \theta\right| \max _{i=1,2}\left\|D_{e_{i}} E\right\|_{L_{\infty}\left(e_{i} \cap \tau^{-}\right)} \lesssim \frac{h_{\tau}}{r(\theta)} \max _{i=1,2}\left\|D_{e_{i}} E\right\|_{L_{\infty}\left(e_{i} \cap \tau^{-}\right)} .
$$

Moreover, since $E\left(x_{3}\right)=0$, we have $\left|E\left(x_{3}+t r_{i}^{-} e_{i}\right)\right| \lesssim t h_{\tau}\left\|D_{e_{i}} E\right\|_{L_{\infty}\left(e_{i} \cap \tau^{-}\right)}$, $i=1,2$, and hence the second term on the right-hand side of (3.38) may also be bounded exactly as in the right-hand side of (3.41). An analogous procedure can be applied to $\partial \widetilde{E} / \partial y$, thus yielding, overall,

$$
|\nabla \widetilde{E}(t, \theta)| \lesssim \frac{h_{\tau}}{r(\theta)} \max _{i=1,2}\left\|D_{e_{i}} E\right\|_{L_{\infty}\left(e_{i} \cap \tau^{-}\right)} \quad \text { for } \quad t \in[0,1], \theta \in[0, \beta] .
$$

Therefore, also using (3.39), we obtain the estimate on $\tau^{-}$:

$$
\begin{aligned}
|\widetilde{E}|_{H^{1}\left(\tau^{-}\right), \alpha}^{2}=\int_{\tau_{-}} \widehat{\alpha}|\nabla \widetilde{E}(x, y)|^{2} d x d y & =\widehat{\alpha} \int_{0}^{1} \int_{0}^{\beta}|\nabla \widetilde{E}(t, \theta)|^{2} \operatorname{tr}^{2}(\theta) d \theta d t \\
& \lesssim h_{\tau}^{2} \widehat{\alpha} \max _{i=1,2}\left\|D_{e_{i}} E\right\|_{L_{\infty}\left(e_{i} \cap \tau^{-}\right)}^{2} .
\end{aligned}
$$

Note that we constructed above an explicit expansion $\widetilde{E}$ into $\tau^{-}$whose precise behaviour is quite delicate. For the extension into $\tau^{+}$, it turns out to be sufficient to apply the inverse trace theorem, which only obtains the extension implicitly. Since $\tau^{+}$is a Lipschitz domain, the (inverse) trace theorem (also using (3.34) ) gives an 
extension $\widetilde{E}$ which satisfies ( since $\alpha \lesssim 1$ on $\tau^{+}$)

$$
\begin{aligned}
|\widetilde{E}|_{H^{1}\left(\tau^{+}\right), \alpha}^{2} & \lesssim|\widetilde{E}|_{H^{1}\left(\tau^{+}\right)}^{2} \\
& \lesssim h_{\tau}^{-1}\|\widetilde{E}\|_{L_{2}\left(\partial \tau^{+}\right)}^{2}+h_{\tau}|\widetilde{E}|_{H^{1}\left(\partial \tau^{+}\right)}^{2} \\
= & \sum_{i=1,2,3}\left(h_{\tau}^{-1}\|E\|_{L_{2}\left(e_{i} \cap \tau^{+}\right)}^{2}+h_{\tau}|E|_{H^{1}\left(e_{i} \cap \tau^{+}\right)}^{2}\right) \\
& \quad+h_{\tau}{ }^{-1}\|\widetilde{E}\|_{L_{2}(\Gamma \cap \tau)}^{2}+h_{\tau}|\widetilde{E}|_{H^{1}(\Gamma \cap \tau)}^{2} \\
(3.43) \quad \lesssim & h_{\tau}^{2} \max _{i=1,2,3}\left\|D_{e_{i}} E\right\|_{L_{\infty}\left(e_{i} \cap \tau^{+}\right)}^{2}+h_{\tau}{ }^{-1}\|\widetilde{E}\|_{L_{2}(\Gamma \cap \tau)}^{2}+h_{\tau}|\widetilde{E}|_{H^{1}(\Gamma \cap \tau)}^{2},
\end{aligned}
$$

where in the final estimate we used $E\left(x_{1}\right)=0=E\left(x_{2}\right)$.

It remains to estimate the final two terms in (3.43). First note that on $\Gamma \cap \tau$ we can write $\widetilde{E}=\widetilde{E}(1, \theta)$ for some $\theta \in[0, \beta]$, and by (3.37) we have (as above)

$$
\|\widetilde{E}\|_{L_{\infty}(\Gamma \cap \tau)} \lesssim \max _{i=1,2}\left|E\left(y_{i}\right)\right| \lesssim h_{\tau} \max _{i=1,2}\left\|D_{e_{i}} E\right\|_{L_{\infty}\left(e_{i} \cap \tau^{+}\right)} .
$$

Moreover, writing $\theta=\theta(s)$, where $s$ denotes arclength along $\Gamma \cap \tau$, we have

$$
\left|\frac{d}{d s}\{\widetilde{E}(1, \theta(s))\}\right|=\frac{1}{\beta}\left|E\left(y_{2}\right)-E\left(y_{1}\right)\right|\left|\frac{d \theta}{d s}\right| \lesssim h_{\tau} \max _{i=1,2}\left\|D_{e_{i}} E\right\|_{L_{\infty}\left(e_{i} \cap \tau^{+}\right)}\left|\frac{d \theta}{d s}\right| .
$$

Hence, making use of (3.33) and (3.34), we have

$$
\begin{aligned}
|\widetilde{E}|_{H^{1}\left(\Gamma \cap \tau^{+}\right)}^{2} & \lesssim h_{\tau}^{2} \max _{i=1,2}\left\|D_{e_{i}} E\right\|_{L_{\infty}\left(e_{i} \cap \tau^{+}\right)}^{2} \int_{0}^{|\Gamma \cap \tau|}\left|\frac{d \theta}{d s}\right|^{2} d s \\
& \sim h_{\tau} \max _{i=1,2}\left\|D_{e_{i}} E\right\|_{L_{\infty}\left(e_{i} \cap \tau^{+}\right)}^{2} .
\end{aligned}
$$

The lemma follows by insertion of (3.44) and (3.45) into (3.43).

Theorem 3.16. Let $u$ be the solution of (1.2) and suppose $\tau$ is one of the elements which are cut through by the interface $\Gamma$. Then, under Assumption 3.14 ,

$$
\begin{aligned}
\left|E_{h}^{\mathrm{MS}}\right|_{H^{1}(\tau), \alpha}^{2} & \lesssim h_{\tau}^{2} \widehat{\alpha}^{2}\left[|u|_{H^{2}\left(\tau^{-}\right)}^{2}+h_{\tau}|u|_{H^{5 / 2}\left(\tau^{-}\right)}^{2}\right] \\
& +h_{\tau}^{2}\left[|u|_{H^{2}\left(\tau^{+}\right)}^{2}+h_{\tau}|u|_{H^{5 / 2}\left(\tau^{+}\right)}^{2}\right]+h_{\tau}^{2}\|f\|_{L_{2}(\tau)}^{2}
\end{aligned}
$$

Remark 3.17. When $\tau$ is an element which is not intersected by the interface, the estimate (3.46) still holds but the terms in $|u|_{H^{5 / 2}\left(\tau^{ \pm}\right)}$are absent.

Proof. From Theorem 2.2 and Lemma 3.15 we have

$$
\begin{aligned}
& \quad\left|E_{h}^{\mathrm{MS}}\right|_{H^{1}(t \tau), \alpha}^{2} \\
& \lesssim h_{\tau}^{2}\left(\widehat{\alpha} \max _{i=1,2,3}\left\|D_{e_{i}} E_{h}^{\mathrm{MS}}\right\|_{L_{\infty}\left(e_{i} \cap \tau^{-}\right)}^{2}+\max _{i=1,2,3}\left\|D_{e_{i}} E_{h}^{\mathrm{MS}}\right\|_{L_{\infty}\left(e_{i} \cap \tau^{+}\right)}^{2}+\|f\|_{L_{2}(\tau)}^{2}\right) .
\end{aligned}
$$

The edge derivatives on the right-hand side of (3.47) may be estimated by Theorem 3.9, yielding

$$
\begin{aligned}
& \quad\left|E_{h}^{\mathrm{MS}}\right|_{H^{1}(\tau), \alpha}^{2} \\
& \lesssim h_{\tau}^{3} \max _{\substack{i=1,2,3 \\
|k|=1}}\left[\widehat{\alpha}^{2}\left\|D^{k} D_{e_{i}} u\right\|_{L_{2}\left(e_{i} \cap \tau^{-}\right)}^{2}+\left\|D^{k} D_{e_{i}} u\right\|_{L_{2}\left(e_{i} \cap \tau^{+}\right)}^{2}\right]+h_{\tau}^{2}\|f\|_{L_{2}(\tau)}^{2} .
\end{aligned}
$$

Now we adapt the procedure from the proof of Theorem 2.5 to bound the terms in $u$ appearing on the right-hand side of (3.48). Let $\eta^{-}$be a polygon chosen inside 

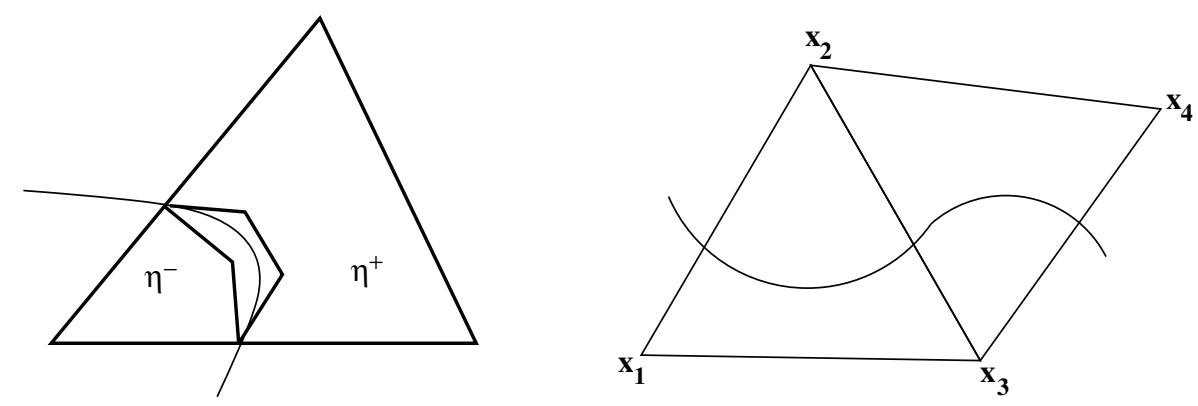

Figure 4. Left: $\Gamma$ is the curved interface and $\eta^{-}\left(\eta^{+}\right)$is the polygon inside $\tau^{-}$(respectively $\tau^{+}$). Right: Two elements intersected by the interface.

$\tau^{-}$with the property that $\partial \tau \cap \tau^{-} \subset \partial \eta^{-}$and let $\eta^{+}$be chosen analogously (see Figure 4, left). Clearly we may choose these polygons so that $\left|\eta^{ \pm}\right| \sim\left|\tau^{ \pm}\right|$.

Then, for $|k|=1$ and $i=1,2$, we have (cf. (2.19) )

$$
\begin{aligned}
\left\|D^{k} D_{e_{i}} u^{-}\right\|_{L_{2}\left(e_{i} \cap \tau^{-}\right)}^{2} & =\left|D^{k} u^{-}\right|_{H^{1}\left(e_{i} \cap \tau^{-}\right)}^{2} \\
& \lesssim h_{\tau}^{-1}\left|D^{k} u^{-}\right|_{H^{1}\left(\eta^{-}\right)}^{2}+\left|D^{k} u^{-}\right|_{H^{3 / 2}\left(\eta^{-}\right)}^{2} \\
& \lesssim h_{\tau}^{-1}\left|u^{-}\right|_{H^{2}\left(\tau^{-}\right)}^{2}+\left|u^{-}\right|_{H^{5 / 2}\left(\tau^{-}\right)}^{2}
\end{aligned}
$$

Analogously we have

$$
\left\|D^{k} D_{e_{i}} u^{+}\right\|_{L_{2}\left(e_{i} \cap \tau^{+}\right)}^{2} \lesssim h_{\tau}^{-1}\left|u^{+}\right|_{H^{2}\left(\tau^{+}\right)}^{2}+\left|u^{+}\right|_{H^{5 / 2}\left(\tau^{+}\right)}^{2} .
$$

The required result follows by combining (3.49) and (3.50) with (3.48)

3.4. Conforming modification and global error estimate. The multiscale basis functions discussed in the previous sections were obtained by solving (2.1) on each element $\tau$ individually, using a boundary condition relevant to that particular element. When an interface cuts an element edge, there is no guarantee that the boundary condition will match across that edge, and so the basis constructed in this way may be discontinuous (i.e. the element may be non-conforming). However, as we now show, it is easy to make the basis functions continuous by local averaging. Consider the interface crossing an edge belonging to two adjacent elements, as in Figure 4 (right). Let $x_{p}$ denote any one of the nodes of this pair of triangles and denote the boundary condition on $\overline{x_{2} x_{3}}$ (constructed by the method in $\$ 3.2$ ) for $\tau=\triangle x_{1} x_{2} x_{3}$ by $\phi_{p, \tau}$ and the analogous boundary condition for $\tau^{\prime}=\triangle x_{4} x_{2} x_{3}$ by $\phi_{p, \tau^{\prime}}$. Then we simply define the averaged boundary condition on $\overline{x_{2} x_{3}}$ to be

$$
\frac{\left(\phi_{p, \tau}+\phi_{p, \tau^{\prime}}\right)}{2} \text {. }
$$

Doing this for all edges cut by the interface yields a conforming method. Moreover, we can show by a simple application of the triangle inequality that the new boundary condition yields multiscale basis functions and an interpolation operator which satisfies the estimate in Theorem 3.9, and hence Theorem 3.16 remains true. However, the price we pay is that the resulting basis functions may have a slightly larger support than the standard linear functions. For example in Figure 4 (right), when $p=4$, the basis function $\Phi_{p}^{\mathrm{MS}}$ will not necessarily vanish in the triangle $x_{1} x_{2} x_{3}$. 
Theorem 3.18. Suppose Assumption 3.14 holds for each element which is cut through by the interface. Suppose also that $f \in H^{1 / 2}(\Omega)$. Let $u$ be the solution of (1.2). Assume also that $h$ is sufficiently small. Then

$$
\begin{aligned}
\text { (i) }\left|u-u_{h}^{\mathrm{MS}}\right|_{H^{1}(\Omega), \alpha} & \lesssim h\left[h|f|_{H^{1 / 2}(\Omega)}^{2}+\|f\|_{L_{2}(\Omega)}^{2}\right]^{1 / 2}, \\
\text { (ii) } \quad\left\|u-u_{h}^{\mathrm{MS}}\right\|_{L_{2}(\Omega)} & \lesssim h^{2}\left[h|f|_{H^{1 / 2}(\Omega)}^{2}+\|f\|_{L_{2}(\Omega)}^{2}\right]^{1 / 2} .
\end{aligned}
$$

Proof. Consider first Case I (see (1.5)). By the optimality of $u^{\mathrm{MS}}$ in the energy norm, we get

$$
\left|u-u_{h}^{\mathrm{MS}}\right|_{H^{1}(\Omega), \alpha}^{2} \leq\left|E_{h}^{\mathrm{MS}}\right|_{H^{1}(\Omega), \alpha}^{2}=\sum_{\tau}\left|E_{h}^{\mathrm{MS}}\right|_{H^{1}(\tau), \alpha}^{2} .
$$

We now employ Theorem 3.16 to estimate the right-hand side. Bearing in mind the regularity estimates in Theorem B.1 (since $\partial \Omega$ is assumed to be a convex polygon), we need to assume here that $h$ is small enough so that all elements which cross $\Gamma$ are separated from $\partial \Omega$ by at least a fixed distance. (For example, $h \approx \operatorname{dist}(\Gamma, \partial \Omega) / 2$ would be sufficient.) Under this assumption, and bearing in mind Remark 3.17 we have

$$
\begin{aligned}
& \text { (3.54) } \quad\left|u-u_{h}^{\mathrm{MS}}\right|_{H^{1}(\Omega), \alpha}^{2} \\
& \lesssim h^{2}\left\{\widehat{\alpha}^{2} \sum_{i=1}^{m}\left(|u|_{H^{2}\left(\Omega_{i}\right)}^{2}+h|u|_{H^{5 / 2}\left(\Omega_{i}\right)}^{2}\right)+|u|_{H^{2}\left(\Omega_{0}\right)}^{2}+h|u|_{H^{5 / 2}\left(\Omega_{0}\right)}^{2}+\|f\|_{L_{2}(\Omega)}^{2}\right\} .
\end{aligned}
$$

The required estimate (3.51) follows from the regularity theorem, Theorem B.1. The estimate (3.52) is derived by adapting the duality argument used in the proof of Theorem 2.5 The proof of Case II similar.

Remark 3.19. If $u$ is required to satisfy an inhomogeneous boundary condition $g$ (as described in Remark B.3), then we have the following estimates for Case I:

(i) $\left|u-u_{h}^{\mathrm{MS}}\right|_{H^{1}(\Omega), \alpha} \lesssim h\left[\|f\|_{L_{2}(\Omega)}^{2}+\|g\|_{H^{2}\left(\Omega_{0}\right)}^{2}+h\left(|f|_{H^{1 / 2}(\Omega)}^{2}+|g|_{H^{5 / 2}\left(\Omega_{0}\right)}^{2}\right)\right]^{1 / 2}$,

(ii) $\quad\left\|u-u_{h}^{\mathrm{MS}}\right\|_{L_{2}(\Omega)} \lesssim h^{2}\left[\|f\|_{L_{2}(\Omega)}^{2}+\|g\|_{H^{2}\left(\Omega_{0}\right)}^{2}+h\left(|f|_{H^{1 / 2}(\Omega)}^{2}+|g|_{H^{5 / 2}\left(\Omega_{0}\right)}^{2}\right)\right]^{1 / 2}$,

and, for Case II,

(i) $\quad\left|u-u_{h}^{\mathrm{MS}}\right|_{H^{1}(\Omega), \alpha}$

$$
\lesssim h\left[\|f\|_{L_{2}(\Omega)}^{2}+\widehat{\alpha}^{2}\|g\|_{H^{2}\left(\Omega_{0}\right)}^{2}+h\left(|f|_{H^{1 / 2}(\Omega)}^{2}+\widehat{\alpha}^{2}|g|_{H^{5 / 2}\left(\Omega_{0}\right)}^{2}\right)\right]^{1 / 2}
$$

(ii) $\quad\left\|u-u_{h}^{\mathrm{MS}}\right\|_{L_{2}(\Omega)}$

$$
\lesssim h^{2}\left[\|f\|_{L_{2}(\Omega)}^{2}+\widehat{\alpha}^{2}\|g\|_{H^{2}\left(\Omega_{0}\right)}^{2}+h\left(|f|_{H^{1 / 2}(\Omega)}^{2}+\widehat{\alpha}^{2}|g|_{H^{5 / 2}\left(\Omega_{0}\right)}^{2}\right)\right]^{1 / 2},
$$

The latter estimates can be pessimistic in some inhomogeneous Dirichlet cases. For example, if $u=u_{0}+C$ where $u_{0}$ enjoys the same estimates as in Theorem B.1. then since $\mathcal{I}_{h}^{\mathrm{MS}}$ preserves constants (see (2.5)),

$$
\left|u-u_{h}^{\mathrm{MS}}\right|_{H^{1}(\Omega), \alpha} \leq\left|u-\mathcal{I}_{h}^{\mathrm{MS}} u\right|_{H^{1}(\Omega), \alpha}=\left|u_{0}-\mathcal{I}_{h}^{\mathrm{MS}} u_{0}\right|_{H^{1}(\Omega), \alpha},
$$

and the results of Theorem 3.18 are still valid in this case. 


\section{NUMERICAL EXPERIMENTS}

In this section, we perform three numerical experiments to verify the convergence rates established above. We consider the weak form of the Dirichlet boundary value problem:

$$
-\nabla \cdot(\alpha \nabla u)=f \quad \text { in } \quad \Omega, \quad \text { with } \quad u=g \quad \text { on } \partial \Omega,
$$

for different domains $\Omega$, piecewise constant function $\alpha$, source term $f$ and boundary condition $g$. To compute the multiscale basis functions, we subdivide each coarse grid element into $M$ uniform triangular sub-elements and apply the IFE method of 28 for basis function calculation on each coarse element. As discussed in Remark 3.10, this involves approximating the interface by a straight line in each element of the fine grid which it intersects. Clearly, extending the theoretical results in this paper to this case will require $M$ sufficiently large relative to $h$ and $\alpha$. In the following three experiments, we use $M=1024$ to ensure the errors in computing the basis functions are small. At the end of this section we study in more detail how the choice of $M$ affects the overall error in the method.

Experiment 1. In this experiment, $\Omega=[-1,1] \times[-1,1], f=-9 r$, $g=\frac{r^{3}}{\alpha_{0}}+\left(\frac{1}{\alpha_{1}}-\frac{1}{\alpha_{0}}\right) r_{0}^{3}$, and

$$
\alpha= \begin{cases}\alpha_{1}, & r<r_{0} \\ \alpha_{0}, & r \geq r_{0}\end{cases}
$$

where $r=\left(x^{2}+y^{2}\right)^{1 / 2}$ and $r_{0}=\pi / 6.28$ (see also [28]). The exact solution is

$$
u(r, \theta)= \begin{cases}\frac{r^{3}}{\alpha_{1}}, & r<r_{0}, \\ \frac{r^{3}}{\alpha_{0}}+\left(\frac{1}{\alpha_{1}}-\frac{1}{\alpha_{0}}\right) r_{0}^{3}, & r \geq r_{0} .\end{cases}
$$

Recalling (1.5) and (1.6) we shall study Case I: $\alpha_{1}=\widehat{\alpha}, \alpha_{0}=1$ and Case II: $\alpha_{1}=1, \alpha_{0}=\widehat{\alpha}$. Notice that the source term $f$ is independent of $\widehat{\alpha}$. Although this is an inhomogeneous Dirichlet problem, the general estimates in Remarks 3.19 and B.3 are overly pessimistic for Case II here. In Case II, the exact solution (4.3) satisfies $|u|_{H^{s+2}\left(\Omega_{0}\right)}=\frac{1}{\hat{\alpha}}\left|r^{3}\right|_{H^{s+2}\left(\Omega_{0}\right)}$ and $|u|_{H^{s+2}\left(\Omega_{1}\right)}=\left|r^{3}\right|_{H^{s+2}\left(\Omega_{1}\right)}$ for any $s \geq 0$, which is sufficient for $\widehat{\alpha}$-robust optimal convergence. Thus our method still enjoys the error estimates of Theorem 3.18 in both Cases II and I in this example.

The coarse grid in this case is a uniform triangular grid on $\Omega$. We depict the numerical solutions for both cases in Figure 5. The solutions are flat in the region where the coefficient $\alpha$ is high. Figure $[$, shows that the errors are small but are concentrated along the interface. The errors are presented in Tables 1 and 2, These show that the method is first order in the $H^{1}$ semi-norm and second order in the $L_{2}$ norm as predicted by the theory. (Throughout, we use least squares fitting to estimate the convergence rates.) The independence of $\widehat{\alpha}$ can be observed from Figure 7 .

For this experiment our new multiscale finite element method gives much better performance than the standard linear finite element method (on the same grid). The improvement is more significant when $\widehat{\alpha}$ is very large, which may be expected since we have proved that our multiscale method converges independently of $\widehat{\alpha}$, whereas the asymptotic constant in the error estimate for the standard finite element method may depend on $\widehat{\alpha}$. For example, when $\widehat{\alpha}=10^{5}$, the multiscale finite element method has an $L_{2}$ norm error about 66 times smaller than that of the standard 


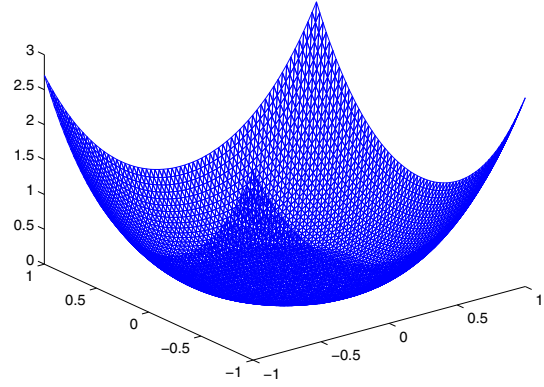

(a) $u_{h}^{\mathrm{MS}}$ for Case I with $\widehat{\alpha}=100000$.

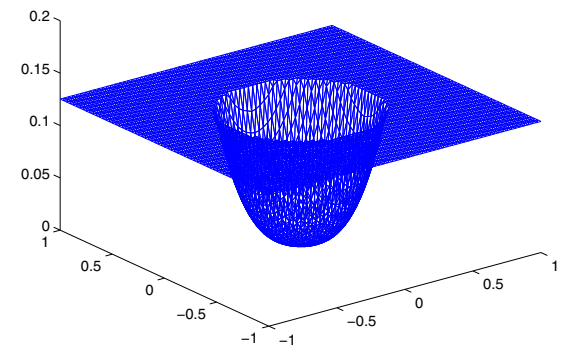

(b) $u_{h}^{\mathrm{MS}}$ for Case II with $\widehat{\alpha}=100000$.

Figure 5. Numerical solutions $u_{h}^{\mathrm{MS}}$ with $h=1 / 32$ for Experiment 1.

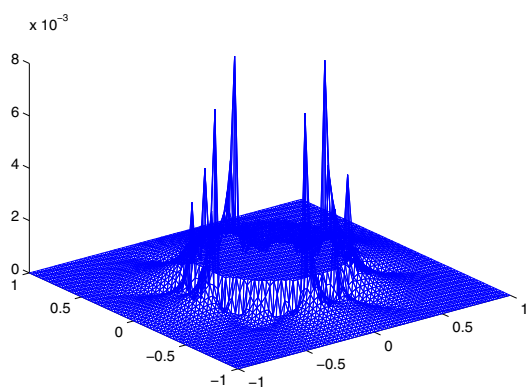

(a) $\left|E_{h}^{\mathrm{MS}}\right|$ for Case I with $\widehat{\alpha}=100000$.

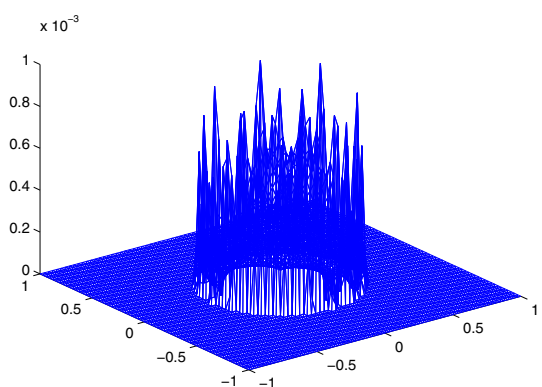

(b) $\left|E_{h}^{\mathrm{MS}}\right|$ for Case II with $\widehat{\alpha}=100000$.

Figure 6. Pointwise errors $E_{h}^{\mathrm{MS}}$ and $h=1 / 32$ for Experiment 1 .

linear finite element method, while in the $H^{1}$ semi-norm the error is better by a factor of about 15. We have also compared our multiscale finite element method with the IFE method [28] applied on the coarse grid, and we found that our method gives a consistently better performance for all values of $\widehat{\alpha}$, although the gain is less pronounced compared with that of the standard finite element method.

Experiment 2. In this experiment $\Omega$ is the unit disk, $\alpha$ is as defined in (4.2), with $r_{0}=1 / 3, f=0$ and $g(x)=x$. The exact solution can be obtained analytically:

$$
u(x, y)= \begin{cases}\frac{-2}{(\beta-1) r_{0}^{2}-(\beta+1)} x, & r<0, \\ \frac{-(\beta+1)}{(\beta-1) r_{0}^{2}-(\beta+1)} x+\frac{(\beta-1) r_{0}^{2}}{(\beta-1) r_{0}^{2}-(\beta+1)} \frac{x}{x^{2}+y^{2}}, & r \geq r_{0},\end{cases}
$$

where $\beta=\alpha_{1} / \alpha_{0}$. Unlike Experiment 1, the exact solution depends on the polar angle $\theta$. We investigate convergence for the case $\alpha_{1}=\widehat{\alpha}, \alpha_{0}=1$, with increasing $\widehat{\alpha}$ (i.e. Case I), using quasi-uniform meshes, with a typical example shown in Figure 8. A typical numerical solution and pointwise error are shown in Figure 9. As in Experiment 1, we can see that the solution is flat in the high conductivity region and that the errors are small and concentrated along the interface. From Table 3 . 
TABLE 1 . The $L_{2}$-norm errors (upper) and the $H^{1}$ semi-norm errors (lower) for the Case I: $\alpha_{1}=\widehat{\alpha}, \alpha_{0}=1$ in Experiment 1.

\begin{tabular}{|c|c|c|c|c|c|}
\hline$h$ & $\hat{\alpha}=10$ & $\hat{\alpha}=100$ & $\hat{\alpha}=1000$ & $\hat{\alpha}=10000$ & $\hat{\alpha}=100000$ \\
\hline $1 / 4$ & $8.9457 \mathrm{e}-2$ & $9.0295 \mathrm{e}-2$ & $8.9569 \mathrm{e}-2$ & $8.9489 \mathrm{e}-2$ & $9.0375 \mathrm{e}-2$ \\
\hline $1 / 8$ & $2.2833 \mathrm{e}-2$ & $2.2877 \mathrm{e}-2$ & $2.2881 \mathrm{e}-2$ & $2.2891 \mathrm{e}-2$ & $2.2912 \mathrm{e}-2$ \\
\hline $1 / 16$ & $5.7666 \mathrm{e}-3$ & $5.7703 \mathrm{e}-3$ & $5.7791 \mathrm{e}-3$ & $5.7824 \mathrm{e}-3$ & $5.7808 \mathrm{e}-3$ \\
\hline $1 / 32$ & $1.4548 \mathrm{e}-3$ & $1.4521 \mathrm{e}-3$ & $1.4511 \mathrm{e}-3$ & $1.4517 \mathrm{e}-3$ & $1.4511 \mathrm{e}-3$ \\
\hline $1 / 64$ & $3.6619 \mathrm{e}-4$ & $3.6242 \mathrm{e}-4$ & $3.6482 \mathrm{e}-4$ & $3.6369 \mathrm{e}-4$ & $3.6366 \mathrm{e}-4$ \\
\hline rate & 1.9837 & 1.9899 & 1.9858 & 1.9865 & 1.9895 \\
\hline
\end{tabular}

\begin{tabular}{|c|c|c|c|c|c|}
\hline$h$ & $\widehat{\alpha}=10$ & $\widehat{\alpha}=100$ & $\widehat{\alpha}=1000$ & $\widehat{\alpha}=10000$ & $\widehat{\alpha}=100000$ \\
\hline $1 / 4$ & $5.1756 \mathrm{e}-1$ & $5.5251 \mathrm{e}-1$ & $5.1793 \mathrm{e}-1$ & $5.2480 \mathrm{e}-1$ & $5.5458 \mathrm{e}-1$ \\
\hline $1 / 8$ & $2.4868 \mathrm{e}-1$ & $2.5246 \mathrm{e}-1$ & $2.4854 \mathrm{e}-1$ & $2.4858 \mathrm{e}-1$ & $2.5381 \mathrm{e}-1$ \\
\hline $1 / 16$ & $1.2349 \mathrm{e}-1$ & $1.2339 \mathrm{e}-1$ & $1.2355 \mathrm{e}-1$ & $1.2297 \mathrm{e}-1$ & $1.2377 \mathrm{e}-1$ \\
\hline $1 / 32$ & $6.2156 \mathrm{e}-2$ & $6.1687 \mathrm{e}-2$ & $6.1456 \mathrm{e}-2$ & $6.1289 \mathrm{e}-2$ & $6.1355 \mathrm{e}-2$ \\
\hline $1 / 64$ & $3.1374 \mathrm{e}-2$ & $3.1011 \mathrm{e}-2$ & $3.0915 \mathrm{e}-2$ & $3.0651 \mathrm{e}-2$ & $3.0662 \mathrm{e}-2$ \\
\hline rate & 1.0088 & 1.0343 & 1.0149 & 1.0216 & 1.0402 \\
\hline
\end{tabular}

TABLE 2. The $L_{2}$-norm errors (upper) and the $H^{1}$ semi-norm errors (lower) for the Case II: $\alpha_{1}=1, \alpha_{0}=\widehat{\alpha}$ in Experiment 1 .

\begin{tabular}{|c|c|c|c|c|c|}
\hline$h$ & $\widehat{\alpha}=10$ & $\widehat{\alpha}=100$ & $\widehat{\alpha}=1000$ & $\widehat{\alpha}=10000$ & $\widehat{\alpha}=100000$ \\
\hline $1 / 4$ & $1.2782 \mathrm{e}-2$ & $9.0781 \mathrm{e}-3$ & $9.3489 \mathrm{e}-3$ & $9.2490 \mathrm{e}-3$ & $9.2439 \mathrm{e}-3$ \\
\hline $1 / 8$ & $3.7991 \mathrm{e}-3$ & $2.8410 \mathrm{e}-3$ & $3.0394 \mathrm{e}-3$ & $2.9212 \mathrm{e}-3$ & $2.9314 \mathrm{e}-3$ \\
\hline $1 / 16$ & $1.0235 \mathrm{e}-3$ & $9.3213 \mathrm{e}-4$ & $9.2752 \mathrm{e}-4$ & $8.3648 \mathrm{e}-4$ & $8.5214 \mathrm{e}-4$ \\
\hline $1 / 32$ & $2.7485 \mathrm{e}-4$ & $2.7843 \mathrm{e}-4$ & $2.4049 \mathrm{e}-4$ & $2.2169 \mathrm{e}-4$ & $2.2716 \mathrm{e}-4$ \\
\hline $1 / 64$ & $7.7605 \mathrm{e}-5$ & $6.6592 \mathrm{e}-5$ & $5.4716 \mathrm{e}-5$ & $5.7664 \mathrm{e}-5$ & $5.9580 \mathrm{e}-5$ \\
\hline rate & 1.8517 & 1.7533 & 1.8493 & 1.8371 & 1.8245 \\
\hline
\end{tabular}

\begin{tabular}{|c|c|c|c|c|c|}
\hline$h$ & $\widehat{\alpha}=10$ & $\widehat{\alpha}=100$ & $\widehat{\alpha}=1000$ & $\widehat{\alpha}=10000$ & $\widehat{\alpha}=100000$ \\
\hline $1 / 4$ & $1.3950 \mathrm{e}-1$ & $1.2346 \mathrm{e}-1$ & $1.2486 \mathrm{e}-1$ & $1.2422 \mathrm{e}-1$ & $1.2408 \mathrm{e}-1$ \\
\hline $1 / 8$ & $6.7497 \mathrm{e}-2$ & $5.7930 \mathrm{e}-2$ & $5.7251 \mathrm{e}-2$ & $5.7320 \mathrm{e}-2$ & $5.7267 \mathrm{e}-2$ \\
\hline $1 / 16$ & $3.3704 \mathrm{e}-2$ & $3.0806 \mathrm{e}-2$ & $2.6738 \mathrm{e}-2$ & $2.6893 \mathrm{e}-2$ & $2.6961 \mathrm{e}-2$ \\
\hline $1 / 32$ & $1.8304 \mathrm{e}-2$ & $1.4854 \mathrm{e}-2$ & $1.2806 \mathrm{e}-2$ & $1.2563 \mathrm{e}-2$ & $1.2609 \mathrm{e}-2$ \\
\hline $1 / 64$ & $9.9543 \mathrm{e}-3$ & $7.3327 \mathrm{e}-3$ & $6.2600 \mathrm{e}-3$ & $6.0577 \mathrm{e}-3$ & $6.2529 \mathrm{e}-3$ \\
\hline rate & 0.9987 & 0.9708 & 0.9982 & 1.0063 & 1.0160 \\
\hline
\end{tabular}

we can see that the convergence rates are very close to optimal and independent of $\widehat{\alpha}$. Although the theory presented above is for polygonal $\partial \Omega$, the error estimates in Remark 3.19 could easily be extended to prove $\widehat{\alpha}$-independent convergence of optimal order for this experiment, as is observed in the tables. 


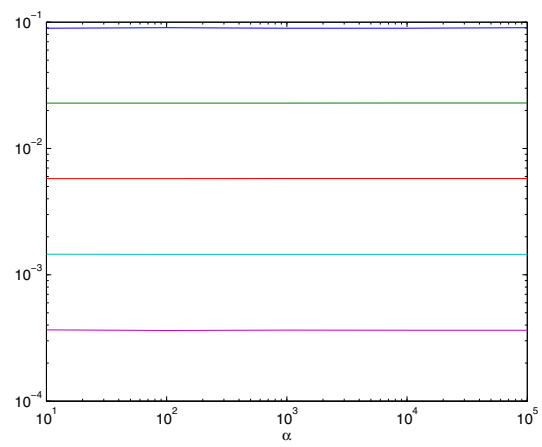

(a) $L_{2}$-norm error for Case I.

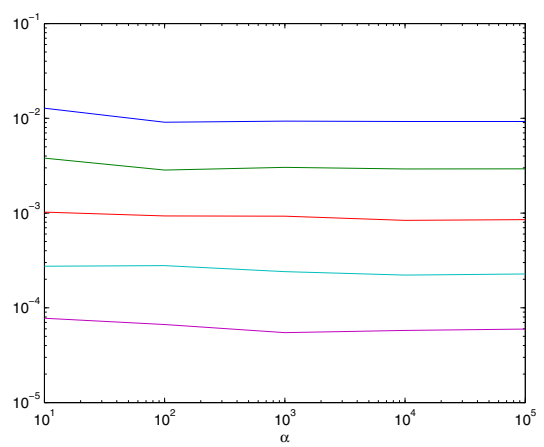

(c) $L_{2}$-norm error for Case II.

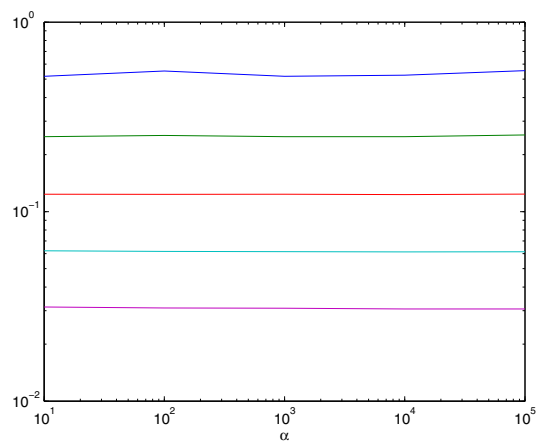

(b) $H^{1}$ semi-norm error for Case I.

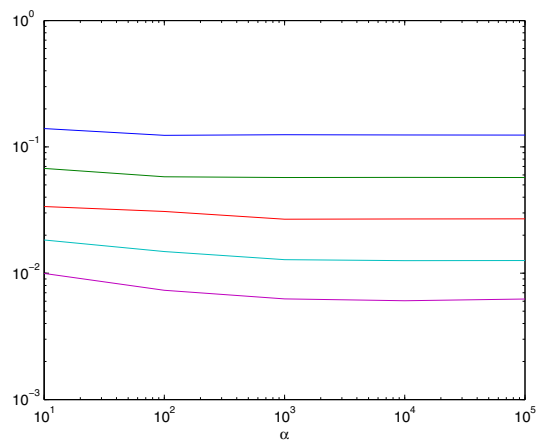

(d) $H^{1}$ semi-norm error for Case II.

Figure 7. Figures (a)-(d) show that the errors are not affected by the values of $\widehat{\alpha}$ in Experiment 1. Each line represents the error versus $\widehat{\alpha}$ for fixed $h$. The values of $h$ are $1 / 4,1 / 8,1 / 16,1 / 32,1 / 64$ from top to bottom.

TABLE 3 . The $L_{2}$-norm errors (upper) and the $H^{1}$ semi-norm errors (lower) for Experiment 2.

\begin{tabular}{|c|c|c|c|c|c|}
\hline$h$ & $\widehat{\alpha}=10$ & $\widehat{\alpha}=100$ & $\widehat{\alpha}=1000$ & $\widehat{\alpha}=10000$ & $\widehat{\alpha}=100000$ \\
\hline $1 / 8$ & $2.2893 \mathrm{e}-3$ & $4.6732 \mathrm{e}-3$ & $3.4460 \mathrm{e}-3$ & $3.3769 \mathrm{e}-3$ & $3.3855 \mathrm{e}-3$ \\
\hline $1 / 16$ & $7.0721 \mathrm{e}-4$ & $1.7751 \mathrm{e}-3$ & $9.0811 \mathrm{e}-4$ & $8.8256 \mathrm{e}-4$ & $8.8731 \mathrm{e}-4$ \\
\hline $1 / 32$ & $1.8442 \mathrm{e}-4$ & $3.1863 \mathrm{e}-4$ & $2.5463 \mathrm{e}-4$ & $2.4886 \mathrm{e}-4$ & $2.8548 \mathrm{e}-4$ \\
\hline $1 / 64$ & $5.2058 \mathrm{e}-5$ & $7.9585 \mathrm{e}-5$ & $7.0451 \mathrm{e}-5$ & $7.0448 \mathrm{e}-5$ & $7.0659 \mathrm{e}-5$ \\
\hline rate & 1.8315 & 2.0105 & 1.8671 & 1.8575 & 1.8383 \\
\hline
\end{tabular}

\begin{tabular}{|c|c|c|c|c|c|}
\hline$h$ & $\widehat{\alpha}=10$ & $\widehat{\alpha}=100$ & $\widehat{\alpha}=1000$ & $\widehat{\alpha}=10000$ & $\widehat{\alpha}=100000$ \\
\hline $1 / 8$ & $7.3816 \mathrm{e}-2$ & $9.1749 \mathrm{e}-2$ & $9.0765 \mathrm{e}-2$ & $9.1247 \mathrm{e}-2$ & $9.1505 \mathrm{e}-2$ \\
\hline $1 / 16$ & $4.1501 \mathrm{e}-2$ & $4.6103 \mathrm{e}-2$ & $4.5586 \mathrm{e}-2$ & $4.5827 \mathrm{e}-2$ & $4.5973 \mathrm{e}-2$ \\
\hline $1 / 32$ & $2.2267 \mathrm{e}-2$ & $2.4132 \mathrm{e}-2$ & $2.3906 \mathrm{e}-2$ & $2.3967 \mathrm{e}-2$ & $2.4874 \mathrm{e}-2$ \\
\hline $1 / 64$ & $1.3250 \mathrm{e}-2$ & $1.3547 \mathrm{e}-2$ & $1.2411 \mathrm{e}-2$ & $1.2333 \mathrm{e}-2$ & $1.2382 \mathrm{e}-2$ \\
\hline rate & 0.8332 & 0.9213 & 0.9543 & 0.9597 & 0.9543 \\
\hline
\end{tabular}




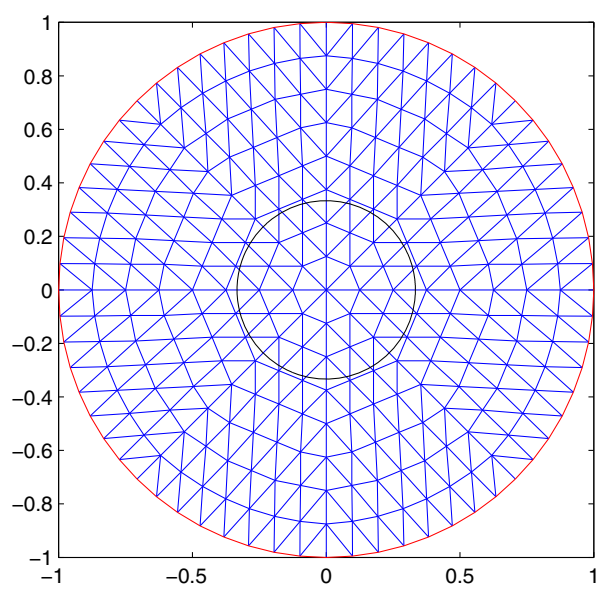

Figure 8. Illustration of computation domain and meshes for Experiment 2. The black circle indicates the interface in the problem.

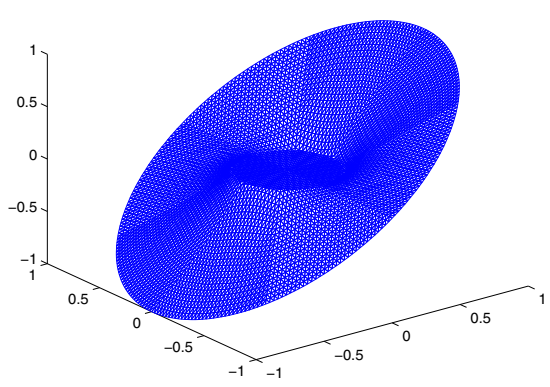

(a) $u_{h}^{\mathrm{MS}}$

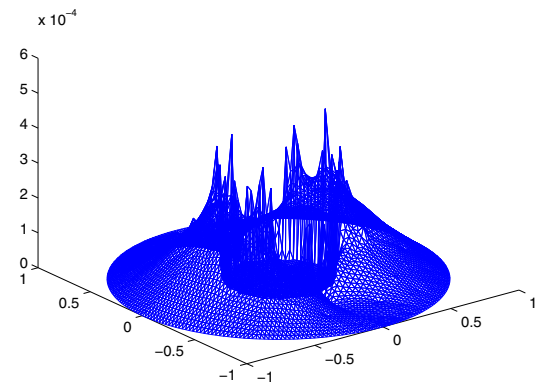

(b) $\left|E_{h}^{\mathrm{MS}}\right|$

Figure 9. Numerical solution $u_{h}^{\mathrm{MS}}$ and pointwise error $E_{h}^{\mathrm{MS}}$ for Experiment 2 with $\widehat{\alpha}=100000$ and $h=1 / 32$.

Experiment 3. In this experiment $\Omega=[-1,1] \times[-1,1]$, and we consider the case of two inclusions with

$$
\alpha= \begin{cases}\alpha_{1}, & \text { when }\left(x-x_{1}\right)^{2}+\left(y-y_{1}\right)^{2}<r_{1}^{2}, \\ \alpha_{2}, & \text { when }\left(x-x_{2}\right)^{2}+\left(y-y_{2}\right)^{2}<r_{2}^{2}, \\ \alpha_{0}, & \text { otherwise }\end{cases}
$$

Here we choose $f=1$ and $g=0$. Since an analytical solution is unknown, we use the solution on the finest mesh (here with 16641 grid points) as the reference solution to compute the error for solutions on coarser meshes. We choose $\left(x_{1}, y_{1}\right)=$ $(1 / 2,0),\left(x_{2}, y_{2}\right)=(-1 / 2,1 / 2)$ and $r_{1}=2 / 5, r_{2}=1 / 3$.

Recalling that (1.5) allows $\alpha_{1}$ and $\alpha_{2}$ to approach infinity with different rates, we set $\alpha_{1}=\widehat{\alpha}, \alpha_{2}=5 \widehat{\alpha}$ and $\alpha_{0}=1$ as an example of Case I. For Case II, we let 


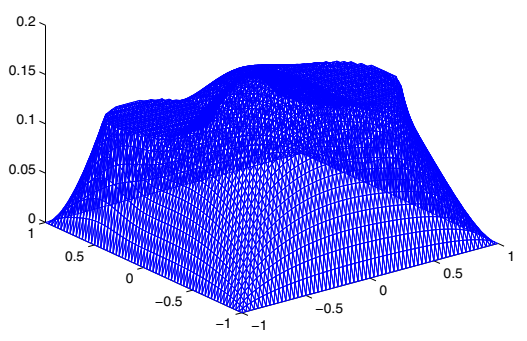

(a) $u_{h}^{\mathrm{MS}}$ for Case I with $\widehat{\alpha}=100000$.

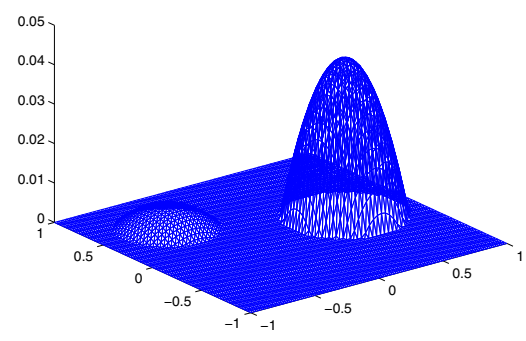

(b) $u_{h}^{\mathrm{MS}}$ for Case II with $\widehat{\alpha}=100000$.

Figure 10. Numerical solutions $u_{h}^{\mathrm{MS}}$ for Experiment 3.

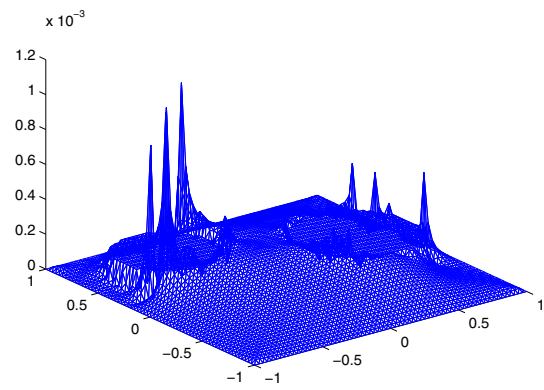

(a) $\left|E_{h}^{\mathrm{MS}}\right|$ for Case I with $\widehat{\alpha}=100000$.

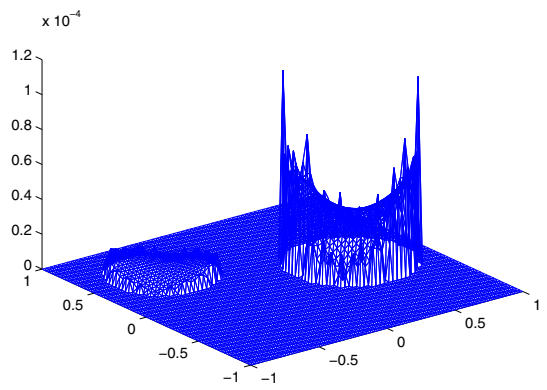

(b) $\left|E_{h}^{\mathrm{MS}}\right|$ for Case II with $\widehat{\alpha}=100000$.

Figure 11. Pointwise errors $E_{h}^{\mathrm{MS}}$ for Experiment 3.

$\alpha_{1}=1, \alpha_{2}=5$ and $\alpha_{0}=\widehat{\alpha}$. The numerical solution and pointwise error are shown in Figure 10 and Figure11. We can see that the error is still small and concentrated along the interface. Tables 4 and 5 show that our method enjoys a roughly optimal convergence rate and that the errors are independent of $\widehat{\alpha}$ as predicted by Theorem 3.18 .

Discussion on the choice of the number of subgrid elements. Finally we discuss the sensitivity of the error in the overall multiscale method to the choice of $M$ (the number of subgrid elements) used to compute the basis functions. Here we test how the errors depend on $M$ in Experiment 1 (Case I), with $\widehat{\alpha}=100,1000,10000$, and $h=1 / 4,1 / 8,1 / 16,1 / 32,1 / 64$, and we plot the error against $M$ in Figure 12 . In each plot there are five groups of three lines, where each group corresponds to a different value of $h$, with $h$ decreasing from $1 / 4$ (top group) to 1/64 (bottom group). In each group of three lines, the dash $*$ line is for $\widehat{\alpha}=100$, the dash $\mathrm{x}$ line is for $\widehat{\alpha}=1000$ and the dash diamond line is for $\widehat{\alpha}=10000$. (In the case of the $L_{2}$ error, the cases $h=1 / 32$ and $1 / 64$ are almost coincidental.) 
TABLE 4 . The $L_{2}$-norm errors (upper) and the $H^{1}$ semi-norm errors (lower) for Case I: $\alpha_{1}=\widehat{\alpha}, \alpha_{2}=5 \widehat{\alpha}, \alpha_{0}=1$ in Experiment 3 .

\begin{tabular}{|c|c|c|c|c|c|}
\hline$h$ & $\widehat{\alpha}=10$ & $\widehat{\alpha}=100$ & $\widehat{\alpha}=1000$ & $\widehat{\alpha}=10000$ & $\widehat{\alpha}=100000$ \\
\hline $1 / 4$ & $2.2150 \mathrm{e}-2$ & $7.2008 \mathrm{e}-3$ & $7.0956 \mathrm{e}-3$ & $7.2309 \mathrm{e}-3$ & $8.1280 \mathrm{e}-3$ \\
\hline $1 / 8$ & $2.9498 \mathrm{e}-3$ & $2.5855 \mathrm{e}-3$ & $2.5863 \mathrm{e}-3$ & $2.6387 \mathrm{e}-3$ & $3.1500 \mathrm{e}-3$ \\
\hline $1 / 16$ & $1.0142 \mathrm{e}-3$ & $7.0168 \mathrm{e}-4$ & $7.1187 \mathrm{e}-4$ & $7.5761 \mathrm{e}-4$ & $1.1063 \mathrm{e}-3$ \\
\hline $1 / 32$ & $1.6523 \mathrm{e}-4$ & $1.9426 \mathrm{e}-4$ & $1.5366 \mathrm{e}-4$ & $1.5484 \mathrm{e}-4$ & $1.7776 \mathrm{e}-4$ \\
\hline rate & 2.2740 & 1.7518 & 1.8449 & 1.8436 & 1.8054 \\
\hline
\end{tabular}

\begin{tabular}{|c|c|c|c|c|c|}
\hline$h$ & $\widehat{\alpha}=10$ & $\widehat{\alpha}=100$ & $\widehat{\alpha}=1000$ & $\widehat{\alpha}=10000$ & $\widehat{\alpha}=100000$ \\
\hline $1 / 4$ & $1.0715 \mathrm{e}-1$ & $3.5010 \mathrm{e}-2$ & $3.5559 \mathrm{e}-2$ & $3.5590 \mathrm{e}-2$ & $3.5765 \mathrm{e}-2$ \\
\hline $1 / 8$ & $2.2953 \mathrm{e}-2$ & $1.5407 \mathrm{e}-2$ & $1.5854 \mathrm{e}-2$ & $1.6046 \mathrm{e}-2$ & $1.8413 \mathrm{e}-2$ \\
\hline $1 / 16$ & $1.2119 \mathrm{e}-2$ & $5.9967 \mathrm{e}-3$ & $6.7962 \mathrm{e}-3$ & $7.9550 \mathrm{e}-3$ & $1.6621 \mathrm{e}-2$ \\
\hline $1 / 32$ & $5.8558 \mathrm{e}-3$ & $3.1782 \mathrm{e}-3$ & $2.0319 \mathrm{e}-3$ & $2.2558 \mathrm{e}-3$ & $4.0509 \mathrm{e}-3$ \\
\hline rate & 1.3502 & 1.1746 & 1.3610 & 1.2951 & 0.9574 \\
\hline
\end{tabular}

TABLE 5. The $L_{2}$-norm errors (upper) and the $H^{1}$ semi-norm errors (lower) for Case II: $\alpha_{1}=1, \alpha_{2}=5, \alpha_{0}=\widehat{\alpha}$ in Experiment 3 .

\begin{tabular}{|c|c|c|c|c|c|}
\hline$h$ & $\widehat{\alpha}=10$ & $\widehat{\alpha}=100$ & $\widehat{\alpha}=1000$ & $\widehat{\alpha}=10000$ & $\widehat{\alpha}=100000$ \\
\hline $1 / 4$ & $6.6651 \mathrm{e}-3$ & $1.3188 \mathrm{e}-3$ & $1.2837 \mathrm{e}-3$ & $1.2870 \mathrm{e}-3$ & $1.2343 \mathrm{e}-3$ \\
\hline $1 / 8$ & $7.7081 \mathrm{e}-4$ & $4.9420 \mathrm{e}-4$ & $3.3161 \mathrm{e}-4$ & $3.3314 \mathrm{e}-4$ & $3.2842 \mathrm{e}-4$ \\
\hline $1 / 16$ & $1.8952 \mathrm{e}-4$ & $1.6737 \mathrm{e}-4$ & $6.9212 \mathrm{e}-5$ & $6.9211 \mathrm{e}-5$ & $6.6224 \mathrm{e}-5$ \\
\hline $1 / 32$ & $4.9532 \mathrm{e}-5$ & $5.8600 \mathrm{e}-5$ & $1.4979 \mathrm{e}-5$ & $1.5999 \mathrm{e}-5$ & $1.6192 \mathrm{e}-5$ \\
\hline rate & 2.3240 & 1.5039 & 2.1524 & 2.1257 & 2.1067 \\
\hline
\end{tabular}

\begin{tabular}{|c|c|c|c|c|c|}
\hline$h$ & $\widehat{\alpha}=10$ & $\widehat{\alpha}=100$ & $\widehat{\alpha}=1000$ & $\widehat{\alpha}=10000$ & $\widehat{\alpha}=100000$ \\
\hline $1 / 4$ & $5.9426 \mathrm{e}-2$ & $9.7096 \mathrm{e}-3$ & $8.7966 \mathrm{e}-3$ & $8.7811 \mathrm{e}-3$ & $8.1776 \mathrm{e}-3$ \\
\hline $1 / 8$ & $1.1250 \mathrm{e}-2$ & $6.9732 \mathrm{e}-3$ & $4.4793 \mathrm{e}-3$ & $4.2302 \mathrm{e}-3$ & $4.4192 \mathrm{e}-3$ \\
\hline $1 / 16$ & $4.7793 \mathrm{e}-3$ & $5.0584 \mathrm{e}-3$ & $1.3624 \mathrm{e}-3$ & $1.2180 \mathrm{e}-3$ & $1.1965 \mathrm{e}-3$ \\
\hline $1 / 32$ & $3.0188 \mathrm{e}-3$ & $2.9506 \mathrm{e}-3$ & $6.1242 \mathrm{e}-4$ & $4.0833 \mathrm{e}-4$ & $4.7003 \mathrm{e}-4$ \\
\hline rate & 1.4132 & 0.5618 & 1.3250 & 1.5076 & 1.4248 \\
\hline
\end{tabular}

From these graphs we can see that the errors decrease as $M$ increases, and with $M=64$, the multiscale finite element method gives an error that is comparable to that of using $M=1024$ when the coarse mesh size is less than $1 / 4$ for all $\widehat{\alpha}$. This indicates that, at least in this example, it is possible to use relatively few subgrid elements to compute the basis function with the desired accuracy, for example by choosing $M=64$. We expect that the use of adaptive subgrid elements may lead to further computational saving in computing the multiscale basis function. 


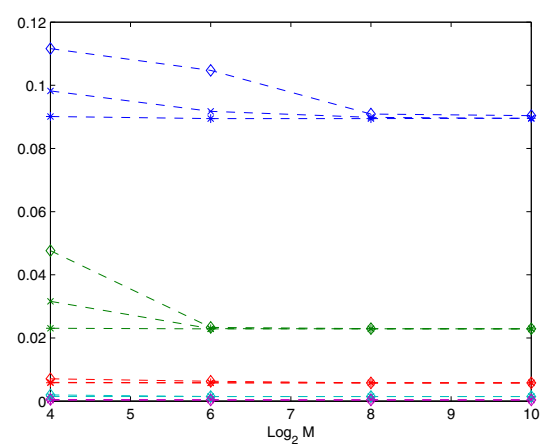

(a) $L_{2}$ norm error versus $M$.

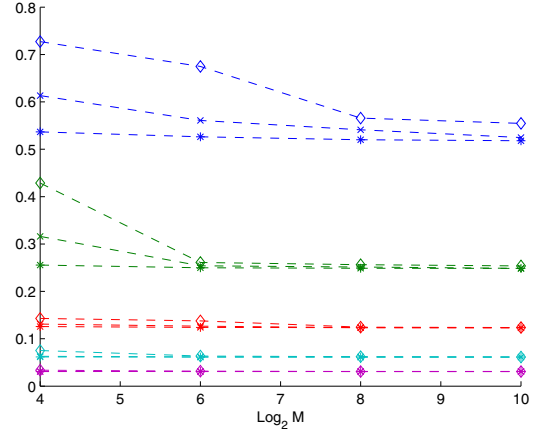

(b) $H^{1}$ semi-norm error versus $M$.

Figure 12. The sensitivity test of $M$ for Case I in Experiment 1 with $\widehat{\alpha}=100(--*$ line $), 1000(--\mathrm{x}$ line $), 10000(--$ diamond line). The values of $h$ are $1 / 4,1 / 8,1 / 16,1 / 32,1 / 64$ from top to bottom.

\section{Appendix A. Extension theorem and Poincaré inequality ON AN ANNULUS}

\section{Lemma A.1. Extension theorem on an annulus.}

Consider an equilateral triangle denoted $\tau$, with side 1 depicted in Figure 13, and let $\tau^{\delta}$ be the closed annulus of uniform width $\delta$ bounded by $\Gamma_{1}$ and $\Gamma_{2}$ as pictured. Let $\Gamma:=\Gamma_{1} \cup \Gamma_{2}$ and let $v \in H^{1 / 2}(\Gamma)$. Then $v$ has an extension $\widetilde{v} \in H^{1}\left(\tau^{\delta}\right)$ such that

$$
|\widetilde{v}|_{H^{1}\left(\tau^{\delta}\right)} \leq C \delta^{-1}\|v\|_{H^{1 / 2}(\Gamma)},
$$

where $C$ is a generic constant independent of $\delta$ and $v$.

Proof. For this proof only, we use the notation $A \lesssim B$ to mean that $A \leq C B$ with $C$ independent of $\delta$ and $u$. We use standard tools for Lipschitz domains (as found for example in [34]). Let $\left\{W_{i}\right\}_{i=1}^{N}$ be an overlapping open covering of $\tau^{\delta}$ and let $\left\{\phi_{i}\right\}_{i=1}^{N}$ be a corresponding partition of unity with the properties: (i) Each $W_{i}$ is the intersection of $\tau^{\delta}$ with an open ball of diameter $\lesssim \delta$, and either $W_{i} \cap \Gamma_{1}=\emptyset$ or $W_{i} \cap \Gamma_{2}=\emptyset$; (ii) $\left\|\nabla \phi_{i}\right\|_{L^{\infty}(\tau)} \lesssim \delta^{-1}$; (iii) each $W_{i}$ has non-empty intersection with at most $m W_{j}$ for some number $m$ independent of $\delta$.

Define $\sigma_{i}=W_{i} \cap \Gamma$. By property (i), $\sigma_{i}$ is either a straight line segment or a corner segment of $\Gamma$. Therefore there exists a bijective $H^{1}$ map $F_{i}: \mathbb{R}^{2} \rightarrow \mathbb{R}^{2}$ such that $\widetilde{\sigma}_{i}:=F_{i}\left(\sigma_{i}\right)$ is a subinterval of the $x$-axis in $\mathbb{R}^{2}$ and $F_{i}\left(W_{i}\right)$ is a bounded subset of the upper half plane $\mathbb{R}_{+}^{2}$. Since $v \circ F_{i}^{-1} \in H^{1 / 2}\left(\widetilde{\sigma}_{i}\right)$ and $\widetilde{\sigma}_{i}$ is an open subset of $\mathbb{R}$, by the Extension Theorem (e.g. [34, Theorem A.4]), we can find an extension function $w_{i}$ on $\mathbb{R}$ such that $\left.w_{i}\right|_{\sigma_{i}}=v \circ F_{i}^{-1}$ and

$$
\left\|w_{i}\right\|_{H^{1 / 2}(\mathbb{R})} \lesssim\left\|v \circ F_{i}^{-1}\right\|_{H^{1 / 2}\left(\widetilde{\sigma}_{i}\right)} \lesssim\|v\|_{H^{1 / 2}\left(\sigma_{i}\right)} .
$$

Then, by the (inverse) Trace Theorem on a half-space (e.g. [34, Lemma 3.36]), we can extend $w_{i}$ to a function $\widetilde{w}_{i}$ on the upper half plane $\mathbb{R}_{+}^{2}$ such that $\widetilde{w}_{i} \in H^{1}\left(\mathbb{R}_{+}^{2}\right)$, $\widetilde{w}_{i}(x, 0)=w_{i}(x), x \in \mathbb{R}$, and

$$
\left\|\widetilde{w}_{i}\right\|_{H^{1}\left(\mathbb{R}_{+}^{2}\right)} \lesssim\left\|w_{i}\right\|_{H^{1 / 2}(\mathbb{R})} \lesssim\|v\|_{H^{1 / 2}\left(\sigma_{i}\right)} .
$$




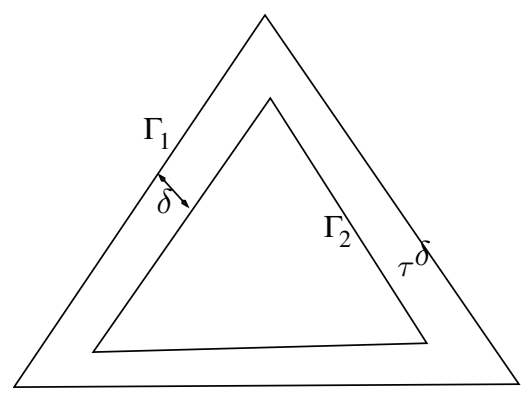

FIGURE 13. The graph of the triangular strip.

Now define $\widetilde{v}_{i}=\widetilde{w}_{i} \circ F_{i}$ and $\widetilde{v}=\sum_{i=1}^{N} \phi_{i} \widetilde{v}_{i}$. Then $\widetilde{v}=v$ on $\Gamma$ and, using property (ii), (iii) and (A.2), we obtain

$$
\begin{aligned}
|\tilde{v}|_{H^{1}\left(\tau^{\delta}\right)}^{2} & \leq \sum_{i=1}^{N}|\tilde{v}|_{H^{1}\left(W_{i}\right)}^{2} \lesssim \sum_{i=1}^{N}\left|\phi_{i} \tilde{v}_{i}\right|_{H^{1}\left(W_{i}\right)}^{2} \lesssim \delta^{-2} \sum_{i=1}^{N}\left\|\tilde{v}_{i}\right\|_{H^{1}\left(W_{i}\right)}^{2} \\
& \lesssim \delta^{-2} \sum_{i=1}^{N}\left\|\tilde{w}_{i}\right\|_{H^{1}\left(\mathbb{R}_{+}^{2}\right)}^{2} \lesssim \delta^{-2} \sum_{i=1}^{N}\|v\|_{H^{1 / 2}\left(\sigma_{i}\right)}^{2} \lesssim \delta^{-2}\|v\|_{H^{1 / 2}(\Gamma)}^{2},
\end{aligned}
$$

as required.

Lemma A.2. Poincaré inequality on an annulus. Let $\tau^{\delta}, \delta$ and $\Gamma=\Gamma_{1} \cup \Gamma_{2}$ be as in Lemma A.1. Then for all $u \in H^{1}\left(\tau^{\delta}\right)$, there exists a constant $\gamma$ such that

$$
\|u-\gamma\|_{L_{2}\left(\tau^{\delta}\right)} \lesssim|u|_{H^{1}\left(\tau^{\delta}\right)}
$$

where the hidden constant is independent of $\delta$.

Proof. Surprisingly, there seems no good source for a proof of this result. However, a proof may be easily constructed by first writing the estimate

$$
\|u-\gamma\|_{L_{2}\left(\tau^{\delta}\right)}^{2} \lesssim \delta\|u-\gamma\|_{L^{2}\left(\Gamma_{2}\right)}^{2}+\delta^{2}|u|_{H^{1}\left(\tau^{\delta}\right)} .
$$

This may be found as equation (3.15) in [41] and can then be combined with the estimate (proved in [36, Lemma 4.3]) that there exists a choice of $\gamma$ which ensures that

$$
\|u-\gamma\|_{L^{2}(\Gamma)}^{2} \lesssim \delta^{-1}|u|_{H^{1}\left(\tau^{\delta}\right)}^{2} .
$$

(To be precise $\gamma$ may be chosen as the average of $u$ over any of the edges of $\tau$.) These two estimates imply the required result.

\section{ApPEndix B. Regularity estimates FOR HIGH-CONTRAST INTERFACE PROBLEMS}

While the Sobolev regularity of the solution $u$ to the interface problem (1.2) is classical, there are relatively few published results which give estimates on how the Sobolev norms of $u$ depend on the contrast parameter $\widehat{\alpha}$. An exception is Huang and Zou (cf. [21]) which gives a partial result in this direction, which we generalise in the following theorem. The proof below was proposed to us by N. Babych, I.V. Kamotski and V.P. Smyshlyaev of the University of Bath, United Kingdom. 
Theorem B.1. Let $\Omega$ be either a smooth $\mathcal{C}^{\infty}$ bounded domain in $\mathbb{R}^{2}$ or a bounded convex polygon, let $\Omega$ contain inclusions $\Omega_{i}, i=1,2 \ldots, m$, each having a $\mathcal{C}^{\infty}$ boundary, and define $\Omega_{0}=\Omega \backslash \bigcup_{i=1}^{m} \bar{\Omega}_{m}$, as described in $₫ 1$. Consider problem (1.2) and assume that either Case I or Case II (1.5) or (1.6) holds. In addition, let $\widetilde{\Gamma}$ denote any closed $\mathcal{C}^{\infty}$ contour in $\Omega_{0}$, which encloses all the $\Omega_{i}$, and let $\widetilde{\Omega}_{0}$ be the domain with boundary $\Gamma \cup \widetilde{\Gamma}$ (see Figure 14 for an illustration in the special case $m=1)$. Then we have

$$
|u|_{H^{s+2}\left(\Omega_{i}\right)} \lesssim \frac{1}{\alpha_{i}}\|f\|_{H^{s}(\Omega)} \quad \text { for all } s \geq 0, i=1,2 \ldots, m .
$$

Moreover,

$$
|u|_{H^{2}\left(\Omega_{0}\right)} \lesssim \frac{1}{\alpha_{0}}\|f\|_{L_{2}(\Omega)}
$$

and

$$
|u|_{H^{2+s}\left(\widetilde{\Omega}_{0}\right)} \lesssim \frac{1}{\alpha_{0}}\|f\|_{H^{s}(\Omega)}, \quad \text { for all } s \geq 0 .
$$

The hidden constants depend on the distance of $\Gamma$ from $\partial \Omega$.

Remark B.2. While the estimates (B.2) and (B.3) can be recovered from the results in [21], the result (B.1) cannot be found there, because [21] works only with estimates of norms and proves $\|u\|_{H^{s+2}\left(\Omega_{i}\right)} \lesssim\|f\|_{H^{s}(\Omega)}, i=1, \ldots, m$. The bounds on the $H^{s+2}$-semi-norms in (B.1) are sharper in Case I and are essential for the analysis in the present paper. In Case II the semi-norms of $u$ on the left-hand side of (B.2) and (B.3) can even be replaced by the corresponding norms. However, only the estimates on the semi-norms are needed in this paper.

Proof. In the proof, we consider only the case when $\Omega$ is a convex polygon. The case of smooth $\partial \Omega$ is simpler. Also, only the case of one inclusion is considered, although the proof for $m>1$ inclusions is similar. Thus the geometry which we consider is illustrated in Figure 14. Also, we shall consider only Case I (1.5), i.e.

$$
\widehat{\alpha}=\alpha_{1} \rightarrow \infty, \quad \alpha_{0}=1 .
$$

As we shall explain at the end of the proof, Case II is easy once the proof for Case $\mathrm{I}$ is clear.

Thus our required result in Case I is, for all $s \geq 0$,

$$
\begin{gathered}
|u|_{H^{s+2}\left(\Omega_{i}\right)} \lesssim \frac{1}{\widehat{\alpha}}\|f\|_{H^{s}(\Omega)}, \quad i=1,2 \ldots, m \\
|u|_{H^{2}\left(\Omega_{0}\right)} \lesssim\|f\|_{L_{2}(\Omega)}, \quad \text { and }|u|_{H^{2+s}\left(\widetilde{\Omega}_{0}\right)} \lesssim\|f\|_{H^{s}(\Omega)} .
\end{gathered}
$$

The result is clear for all $\widehat{\alpha} \in[1, A]$ for some fixed $A$, so we only have to prove (B.5), (B.6) for $\widehat{\alpha}$ sufficiently large.

Before beginning the proof, we recall two classical regularity results for elliptic boundary value problems. Let $s \geq 0$ and let $\phi \in H^{s+3 / 2}(\Gamma)$. Then

$$
\left\{\begin{aligned}
\Delta z & =\omega \text { on } \Omega_{1} \\
z & =\phi \text { on } \Gamma \\
\omega & \in H^{s}\left(\Omega_{1}\right)
\end{aligned}\right\} \Longrightarrow\|z\|_{H^{s+2}\left(\Omega_{1}\right)} \lesssim\|\omega\|_{H^{s}\left(\Omega_{1}\right)}+\|\phi\|_{H^{s+3 / 2}(\Gamma)}
$$

and 


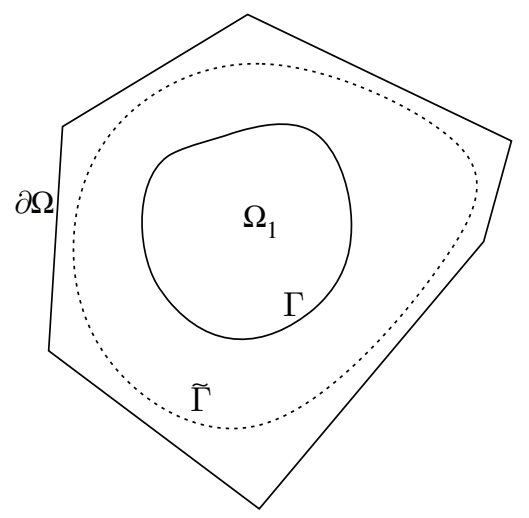

Figure 14 . The domain $\Omega$ in the special case $m=1$. The domain $\Omega_{0}$ is bounded by $\Gamma$ and $\partial \Omega$, while the domain $\widetilde{\Omega}_{0}$ is bounded by $\Gamma$ and $\tilde{\Gamma}$.

(B.8)

$$
\left\{\begin{array}{rllll}
\Delta z & = & \omega & \text { on } & \Omega_{0} \\
z & = & \phi & \text { on } & \Gamma \\
z & = & 0 & \text { on } & \partial \Omega \\
\omega & \in & H^{s}\left(\Omega_{0}\right)
\end{array}\right\} \Longrightarrow\left\{\begin{array}{ll}
\|z\|_{H^{2}\left(\Omega_{0}\right)} \lesssim\|\omega\|_{L_{2}\left(\Omega_{0}\right)}+\|\phi\|_{H^{3 / 2}(\Gamma)} \\
\|z\|_{H^{s+2}\left(\widetilde{\Omega}_{0}\right)} \lesssim\|\omega\|_{H^{s}\left(\Omega_{0}\right)}+\|\phi\|_{H^{s+3 / 2}(\Gamma)}
\end{array}\right\}
$$

A suitable reference for (B.7) is [29, $\S 2$, Thm 5.4]. For the first inequality on the right-hand side of (B.8), we can construct a proof by first extending $\phi$ to a function $\phi^{\prime} \in H^{2}\left(\Omega_{0}\right)$ which vanishes on $\partial \Omega$ and with $\left\|\phi^{\prime}\right\|_{H^{2}\left(\Omega_{0}\right)} \lesssim\|\phi\|_{H^{3 / 2}(\Gamma)}$. Then $\Delta\left(z-\phi^{\prime}\right)=\omega-\Delta \phi^{\prime}$ on $\Omega_{0}$ and $z-\phi^{\prime}$ vanishes on $\partial \Omega_{0}$. So using estimates for elliptic equations on domains with convex corners (for example [17, §3.2]), we obtain the estimate. To obtain the second inequality on the right-hand side of (B.8), we use interior regularity results (a suitable reference is [40, Th 11.1]) and then the trace theorem to obtain

$$
\|z\|_{H^{s+3 / 2}(\tilde{\Gamma})} \lesssim\|\omega\|_{H^{s}\left(\Omega_{0}\right)}+\|z\|_{H^{1}\left(\Omega_{0}\right)} \lesssim\|\omega\|_{H^{s}\left(\Omega_{0}\right)}+\|\phi\|_{H^{1 / 2}(\Gamma)} .
$$

Again applying regularity estimates on the smooth domain $\widetilde{\Omega}_{0}([29, \S 2$, Thm 5.4]), we obtain the required estimate.

Now the first step in the proof is to introduce a decomposition of the form

$$
u=\widehat{u}+\widetilde{u},
$$

where $\widehat{u}$ solves independent Dirichlet problems with homogeneous boundary data on each $\Omega_{i}$ :

$$
-\alpha_{i} \Delta \widehat{u}=f \quad \text { on } \quad \Omega_{i}, \quad \text { with } \quad \widehat{u}=0 \quad \text { on } \quad \partial \Omega_{i}, \quad i=0,1 .
$$

Then, from (B.7) and (B.8) and recalling (B.4), we obtain, for all $s \geq 0$,

$$
\begin{aligned}
& \|\widehat{u}\|_{H^{2+s}\left(\Omega_{1}\right)} \lesssim \frac{1}{\widehat{\alpha}}\|f\|_{H^{s}\left(\Omega_{1}\right)}, \quad\|\widehat{u}\|_{H^{2}\left(\Omega_{0}\right)} \lesssim\|f\|_{L_{2}\left(\Omega_{0}\right)} \\
& \text { and }\|\widehat{u}\|_{H^{2+s}\left(\widetilde{\Omega}_{0}\right)} \lesssim\|f\|_{H^{s}\left(\Omega_{0}\right)} .
\end{aligned}
$$


Thus $\widehat{u}$ satisfies all the estimates (B.5) and (B.6), and the remainder of the proof is concerned with obtaining the same estimates for $\widetilde{u}$. Since $\widetilde{u}=u-\widehat{u}$, it follows that

$$
\Delta \widetilde{u}=0 \quad \text { on } \quad \Omega_{i}, \quad i=0,1, \quad \text { and } \quad \widetilde{u}=0 \quad \text { on } \quad \partial \Omega .
$$

Since $\widetilde{u}$ is continuous across $\Gamma$, we can define $\widetilde{v}=\left.\widetilde{u}\right|_{\Gamma}$.

For any suitably smooth $v$ defined on $\Omega$, we let $\partial v_{i} / \partial n$ denote the normal derivative of $v$ evaluated on $\Gamma$, with value taken from within $\Omega_{i}, i=0,1$. (The normal direction is fixed as outward from $\Omega_{1}$.) Then the usual jump relation for the solution $u$ of the interface problem (1.2) reads: $\left(\partial u_{0} / \partial n\right)-\widehat{\alpha}\left(\partial u_{1} / \partial n\right)=0$, which immediately implies that the function $\widetilde{u}$ satisfies the following equation on $\Gamma$ :

$$
\frac{\partial \widetilde{u}_{0}}{\partial n}-\widehat{\alpha} \frac{\partial \widetilde{u}_{1}}{\partial n}=G:=\widehat{\alpha} \frac{\partial \widehat{u}_{1}}{\partial n}-\frac{\partial \widehat{u}_{0}}{\partial n} .
$$

This may be readily written as

$$
\left(\mathcal{N}_{0}-\widehat{\alpha} \mathcal{N}_{1}\right) \widetilde{v}=G
$$

with $\mathcal{N}_{i}$ denoting appropriate Dirichlet to Neumann maps on $\Omega_{i}$ (taking $\widetilde{v}$ as Dirichlet data on $\Gamma$ and using homogeneous Dirichlet data on $\partial \Omega$ ).

To analyse B.13) as $\widehat{\alpha} \rightarrow \infty$, we might consider scaling by $\widehat{\alpha}^{-1}$ to obtain a small perturbation of $\mathcal{N}_{1}$. However, because $\mathcal{N}_{1}$ has a non-trivial kernel (namely the constant functions on $\Gamma$, henceforth denoted $\langle 1\rangle$ ), we must study the operator $\mathcal{N}_{1}$ in the orthogonal complement of this space. Thus we introduce

$$
\mathcal{P} v=\frac{1}{|\Gamma|} \int_{\Gamma} v(s) d s
$$

the orthogonal projection from $L_{2}(\Gamma)$ onto $\langle 1\rangle$ and $(I-\mathcal{P})$, the orthogonal projection onto $L_{2}(\Gamma)^{\perp}:=\left\{v \in L_{2}(\Gamma): \mathcal{P} v=0\right\}$. Then writing

$$
\widetilde{v}=\mathcal{P} \widetilde{v}+(I-\mathcal{P}) \widetilde{v}=: \widetilde{c}+\widetilde{w},
$$

equation (B.13) may be expressed as the system in $\langle 1\rangle \times L_{2}(\Gamma)^{\perp}$ :

$$
\left[\begin{array}{cc}
\mathcal{P}\left(\mathcal{N}_{0}-\widehat{\alpha} \mathcal{N}_{1}\right) \mathcal{P} & \mathcal{P}\left(\mathcal{N}_{0}-\widehat{\alpha} \mathcal{N}_{1}\right)(I-\mathcal{P}) \\
(I-\mathcal{P})\left(\mathcal{N}_{0}-\widehat{\alpha} \mathcal{N}_{1}\right) \mathcal{P} & (I-\mathcal{P})\left(\mathcal{N}_{0}-\widehat{\alpha} \mathcal{N}_{1}\right)(I-\mathcal{P})
\end{array}\right]\left[\begin{array}{c}
\widetilde{c} \\
\widetilde{w}
\end{array}\right]=\left[\begin{array}{c}
\mathcal{P} G \\
(I-\mathcal{P}) G
\end{array}\right]
$$

Moreover, since $\mathcal{P} \mathcal{N}_{1}=\mathcal{N}_{1} \mathcal{P}$ are null operators on $L_{2}(\Gamma)$, (B.14) may be rewritten as

$$
\left(\mathbf{P}-\widehat{\alpha}^{-1} \mathbf{Q}\right)\left[\begin{array}{c}
\widetilde{c} \\
\widehat{\alpha} \widetilde{w}
\end{array}\right]=\left[\begin{array}{c}
\mathcal{P} G \\
(I-\mathcal{P}) G
\end{array}\right]
$$

where

$$
\mathbf{P}=\left[\begin{array}{cc}
\mathcal{P} \mathcal{N}_{0} \mathcal{P} & 0 \\
(I-\mathcal{P}) \mathcal{N}_{0} \mathcal{P} & -\mathcal{N}_{1}
\end{array}\right] \quad \text { and } \quad \mathbf{Q}=\left[\begin{array}{cc}
0 & \mathcal{P} \mathcal{N}_{0}(I-\mathcal{P}) \\
0 & (I-\mathcal{P}) \mathcal{N}_{0}(I-\mathcal{P})
\end{array}\right]
$$

We next show that $\mathbf{P}$ is invertible on $\langle 1\rangle \times L_{2}(\Gamma)^{\perp}$. Note first that $\mathcal{N}_{1}$ is invertible on $L_{2}(\Gamma)^{\perp}$. To analyse $\mathcal{P} \mathcal{N}_{0} \mathcal{P}$, consider the boundary value problem:

$$
\Delta \eta=0 \text { in } \Omega_{0}, \quad \text { with } \eta=1 \text { on } \Gamma \text { and } \eta=0 \text { on } \partial \Omega,
$$

which has a unique solution $\eta \in H^{2}\left(\Omega_{0}\right)$. The linear operator $\mathcal{P} \mathcal{N}_{0} \mathcal{P}$ operates on $\langle 1\rangle$ as multiplication by the scalar

$$
\gamma:=\mathcal{P}\left[\frac{\partial \eta}{\partial n}\right]=\frac{1}{|\Gamma|} \int_{\Gamma} \frac{\partial \eta}{\partial n} d s
$$


and this scalar does not vanish, since (by (B.16)

$$
\gamma|\Gamma|=\int_{\Gamma} \frac{\partial \eta}{\partial n} d s=\int_{\partial \Omega_{0}} \eta \frac{\partial \eta}{\partial n} d s=\int_{\Omega_{0}} \nabla \cdot(\eta \nabla \eta) d x=\int_{\Omega_{0}}|\nabla \eta|^{2} d x>0 .
$$

Moreover, the linear operator $(I-\mathcal{P}) \mathcal{N}_{0} \mathcal{P}$ operates on $\langle 1\rangle$ as multiplication by the function $\rho:=(I-\mathcal{P})(\partial \eta / \partial n)=\partial \eta / \partial n-\gamma \in L_{2}(\Gamma)^{\perp}$. Hence

$$
\mathbf{P}=\left[\begin{array}{cc}
\gamma & 0 \\
\rho & -\mathcal{N}_{1}
\end{array}\right] \quad \text { and } \quad \mathbf{P}^{-1}=\left[\begin{array}{cc}
\gamma^{-1} & 0 \\
\gamma^{-1} \mathcal{N}_{1}^{-1} \rho & -\mathcal{N}_{1}^{-1}
\end{array}\right] .
$$

Now combining (B.7) and (B.8) with the Trace Theorem we obtain that $\mathcal{N}_{1}$ : $L_{2}(\Gamma)^{\perp} \cap H^{s+3 / 2}(\Gamma) \rightarrow L_{2}(\Gamma)^{\perp} \cap H^{s+1 / 2}(\Gamma)$ is a bounded operator and in fact has a bounded inverse (see, e.g. [29, §2, Th. 5.4]). Moreover, $\mathcal{N}_{0}: H^{s+3 / 2}(\Gamma) \rightarrow$ $H^{s+1 / 2}(\Gamma)$ is also bounded, and it is straightforward to show that $\mathbf{P}^{-1} \mathbf{Q}$ is a bounded operator on $\langle 1\rangle \times H^{s+3 / 2}(\Gamma)$ and that

$$
\left\|\mathbf{P}^{-1}\left[\begin{array}{c}
\mathcal{P} G \\
(I-\mathcal{P}) G
\end{array}\right]\right\|_{\langle 1\rangle \times H^{s+3 / 2}(\Gamma)} \lesssim\|G\|_{H^{s+1 / 2}(\Gamma)} .
$$

Hence, considering (B.15) for $\widehat{\alpha}$ sufficiently large, we have the estimate

$$
\begin{aligned}
\max \left\{|\widetilde{c}|, \widehat{\alpha}\|\widetilde{w}\|_{H^{s+3 / 2}(\Gamma)}\right\} & \lesssim\|G\|_{H^{s+1 / 2}(\Gamma)} \leq \widehat{\alpha}\left\|\frac{\partial \widehat{u}_{1}}{\partial n}\right\|_{H^{s+1 / 2}(\Gamma)}+\left\|\frac{\partial \widehat{u}_{0}}{\partial n}\right\|_{H^{s+1 / 2}(\Gamma)} \\
& \lesssim \widehat{\alpha}\|\widehat{u}\|_{H^{s+2}\left(\Omega_{1}\right)}+\|\widehat{u}\|_{H^{s+2}\left(\widetilde{\Omega}_{0}\right)} \\
\text { (B.18) } & \lesssim\|f\|_{H^{s}(\Omega)},
\end{aligned}
$$

where the last three estimates are obtained from employing the definition of $G$ in (B.12), then the trace theorem and finally (B.10).

Now recall that $\widetilde{u}$ is harmonic on $\Omega_{1}$ and that $\left.\widetilde{u}\right|_{\Gamma}=: \widetilde{v}=\widetilde{c}+\widetilde{w}$, where $\widetilde{c} \in \mathbb{R}$. Hence, if we define $\widetilde{u}_{1}$ on $\Omega_{1}$ by requiring it to be harmonic and to coincide with $\widetilde{w}$ on $\Gamma$, we have, by uniqueness, $\widetilde{u}=\widetilde{c}+\widetilde{u}_{1}$ on $\Omega_{1}$. Thus by using (B.7) and then (B.18), we have, for all $s \geq 0$,

$$
|\widetilde{u}|_{H^{s+2}\left(\Omega_{1}\right)}=\left|\widetilde{u}_{1}\right|_{H^{s+2}\left(\Omega_{1}\right)} \lesssim\|\widetilde{w}\|_{H^{s+3 / 2}(\Gamma)} \lesssim \frac{1}{\widehat{\alpha}}\|f\|_{H^{s}(\Omega)} .
$$

Combining (B.20) with the first inequality in (B.10) (and recalling (B.4) $)$ then yields the first required estimate (B.5). To obtain (B.6), we note that (B.18) implies that $\|\widetilde{v}\|_{H^{s+3 / 2}(\Gamma)} \lesssim\|f\|_{H^{s}(\Omega)}$, and hence the required estimates follow from (B.8).

Finally we remark why the result is easier to prove in Case II, i.e.

$$
\widehat{\alpha}=\alpha_{0} \rightarrow \infty, \quad \alpha_{1}=1 .
$$

In this case the analysis of $\widehat{u}$ is unchanged, but in the analysis of $\widetilde{v}$ we obtain, instead of (B.13), the equation

$$
\left(\widehat{\alpha} \mathcal{N}_{0}-\mathcal{N}_{1}\right) \widetilde{v}=G:=\frac{\partial \widehat{u}_{1}}{\partial n}-\widehat{\alpha} \frac{\partial \widehat{u}_{0}}{\partial n} .
$$

Since $\mathcal{N}_{0}$ is invertible, the estimate for $\widetilde{v}$ can then be obtained by premultiplying this equation by $\widehat{\alpha}^{-1} \mathcal{N}_{0}^{-1}$ and letting $\widehat{\alpha}$ get sufficiently large, without having to go through the projection procedure leading to the system (B.14).

Remark B.3. Here we briefly discuss the case of inhomogeneous Dirichlet conditions. Consider problem (1.2), but replace $u \in H_{0}^{1}(\Omega)$ by the requirement that $u \in H^{1}(\Omega)$ with $u=g$ on $\partial \Omega$. For simplicity assume that $g$ is the restriction to $\partial \Omega$ of a function 
$g \in H^{s+2}\left(\Omega_{0}\right)$, where $g$ has compact support in $\Omega_{0}$ and with $s$ as large as we wish. Assume also that there is only one inclusion $\Omega_{1}$. Then an analogous argument as that in Theorem B.1 can be carried out, but with $\widehat{u}$ now required to satisfy $\widehat{u}=g$ on $\partial \Omega$, so that, in Case I, by (B.8),

$$
\begin{gathered}
\|\widehat{u}\|_{H^{2+s}\left(\Omega_{1}\right)} \lesssim \frac{1}{\widehat{\alpha}}\|f\|_{H^{s}(\Omega)}, \quad \text { and } \\
\|\widehat{u}\|_{H^{2}\left(\Omega_{0}\right)} \lesssim\|f\|_{L_{2}\left(\Omega_{0}\right)}+\|g\|_{H^{2}\left(\Omega_{0}\right)} \quad \text { and }\|\widehat{u}\|_{H^{2+s}\left(\widetilde{\Omega}_{0}\right)} \lesssim\|f\|_{H^{s}\left(\Omega_{0}\right)}+\|g\|_{H^{2+s}\left(\Omega_{0}\right)}
\end{gathered}
$$

The analysis for $\tilde{u}$ is as in (B.18), (B.19) and (B.20), leading to the estimates for $u$ :

$$
\begin{gathered}
|u|_{H^{2+s}\left(\Omega_{1}\right)} \lesssim \frac{1}{\widehat{\alpha}}\left[\|f\|_{H^{s}(\Omega)}+\|g\|_{H^{s+2}\left(\Omega_{0}\right)}\right], \\
|u|_{H^{2}\left(\Omega_{0}\right)} \lesssim\|f\|_{L_{2}(\Omega)}+\|g\|_{H^{2}\left(\Omega_{0}\right)} \quad \text { and }|u|_{H^{2+s}(\widetilde{\Omega})} \lesssim\|f\|_{H^{s}(\Omega)}+\|g\|_{H^{2+s}\left(\Omega_{0}\right)} .
\end{gathered}
$$

Thus the higher semi-norms of $u$ in the inclusions still decay as $\widehat{\alpha} \rightarrow \infty$.

However, in Case II the semi-norms on $\Omega_{0}$ and $\widetilde{\Omega}_{0}$ do not necessarily decay, and the best estimate for general $g$ is

$$
\begin{aligned}
|u|_{H^{2+s}\left(\Omega_{1}\right)} & \lesssim\|f\|_{H^{s}(\Omega)}+\|g\|_{H^{s+2}\left(\Omega_{0}\right)}, \\
|u|_{H^{2}\left(\Omega_{0}\right)} & \lesssim \frac{1}{\widehat{\alpha}}\left\{\|f\|_{L_{2}(\Omega)}+\widehat{\alpha}|g|_{H^{2}\left(\Omega_{0}\right)}\right\} \\
\text { and }|u|_{H^{2+s}\left(\widetilde{\Omega}_{0}\right)} & \lesssim \frac{1}{\widehat{\alpha}}\left\{\|f\|_{H^{s}(\Omega)}+\widehat{\alpha}|g|_{H^{2+s}\left(\Omega_{0}\right)}\right\} .
\end{aligned}
$$

To give more detail of derivation of the final estimate, note that under the stated assumptions on $g$, we have $u-g \in H_{0}^{1}(\Omega)$ and

$$
a(u-g, v)=(f, v)_{L^{2}(\Omega)}+\widehat{\alpha}(\Delta g, v)_{L^{2}\left(\Omega_{0}\right)} .
$$

Then applying Theorem B.1 we have the stated estimate. It could be useful to have only the semi-norms of $g$ on the right-hand side of (B.22): If the given boundary data $g$ has an extension which is "nearly flat", near $\partial \Omega$ then the estimates (B.22)

will be decaying as $\widehat{\alpha} \rightarrow \infty$. This is the case, for example in Experiment 1 (Case II) in 94

\section{REFERENCES}

1. L. Adams, Z.L. Li, The immersed interface/multigrid methods for interface problems, SIAM J. Sci. Comput. 24 (2002), 463-479. MR1951051 (2004f:65109)

2. M. Ainsworth, Robust a posteriori error estimation for nonconforming finite element approximation, SIAM J. Numer. Anal. 42 (2005), 2320-2341. MR2139395(2006j:65331)

3. I. Babuška, The finite element method for elliptic equations with discontinuous coefficients, Computing 5 (1970), 207-213. MR0277119(43:2856)

4. I. Babuška, G. Caloz, and J.E. Osborn, Special finite element methods for a class of second order elliptic problems with rough coefficients, SIAM J. Numer. Anal. 31 (1994), 945-981. MR:1286212 (95g:65146)

5. I. Babuška and J.E. Osborn. Generalized finite element methods: Their performance and their relation to mixed methods, SIAM J. Numer. Anal. 20 (1983), 510-536. MR701094 (84h:65076)

6. J.W. Barrett and C.M. Elliott, Fitted and unfitted finite element methods for elliptic-equations with smooth interfaces, IMA J. Numer. Anal. 7 (1987), 283-300. MR968524 (90a:65231)

7. C. Bernardi and R. Verfürth, Adaptive finite element methods for elliptic equations with non-smooth coefficients, Numer. Math. 85 (2000), 579-608. MR1771781(2001e:65177)

8. I.-L. Chern and Y.-C. Shu, A coupling interface method for elliptic interface problems, J. Comput. Phys. 225 (2007), 2138-2174. MR2349699(2008k:65221)

9. Z. Chen and J. Zou, Finite element methods and their convergence for elliptic and parabolic interface problems, Numer. Math. 79 (1998), 175-202. MR.1622502 (99d:65313) 
10. M. Dryja, On discontinuous Galerkin methods for elliptic problems with discontinuous coefficients, Computational Methods in Applied Mathematics 3 (2003), 76-85. MR 2002258 (2004i:65120)

11. M.A. Dumett and J.P. Keener, An immersed interface method for solving anisotropic elliptic boundary value problems in three dimensions, SIAM J. Sci. Comput. 25 (2003), 348-367. MR 2047209 (2005g:65157)

12. Y. Efendiev and T.Y. Hou, Multiscale Finite Element Methods: Theory and Applications, Springer Publ., February, 2009. MR2477579

13. R.P. Fedkiw, T. Aslam, B, Merriman and S. Osher, A non-oscillatory Eulerian approach to interfaces in multimaterial flows (the ghost fluid method), J. Comput. Phys. 152 (1999), 457-492. MR1699710 (2000c:76061)

14. D.Gilbarg and N.S. Trudinger, Elliptic Partial Differential Equations of Second Order, Springer-Verlag, Berlin, 1977. MR0473443 (57:13109)

15. I.G. Graham, P. Lechner and R. Scheichl, Domain decomposition for multiscale PDEs, Numer. Math. 106 (2007), 589-626. MR 2317926 (2008f:65242)

16. I.G. Graham and R. Scheichl, Robust domain decomposition algorithms for multiscale PDEs, Numerical Methods for Partial Differential Equations 23 (2007), 859-878. MR2326197 (2008c:65367)

17. P. Grisvard, Elliptic Problems in Nonsmooth Domains, Pitman, Boston, 1985. MR.775683 (86m:35044)

18. T.Y. Hou, Z.L. Li, S. Osher and H. Zhao, A hybrid method for moving interface problems with application to the Hele-Shaw flow, J. Comp. Phys. 134 (1997), 236-252. MR 1458828 (98d:76128)

19. T.Y. Hou and X.-H. Wu, A multiscale finite element method for elliptic problems in composite materials and porous media, J. Comp. Phys. 134 (1997), 169-189. MR.1455261(98e:73132)

20. T.Y. Hou, X.-H. Wu and Z. Cai, Convergence of a multiscale finite element method for elliptic problems with rapidly oscillating coefficients, Math. Comp. 68 (1999), 913-943. MR 1642758 (99i:65126)

21. J. Huang and J. Zou, Some new a priori estimates for second-order elliptic and parabolic interface problems, Journal of Differential Equations 184 (2002), 570-586. MR.1929889 (2003h:35026)

22. B.C. Khoo, T.G. Liu and C.W. Wang, The ghost fluid method for compressible gas-water simulation, J. Comput. Phys. 204 (2005), 193-221. MR2121909(2005j:76079)

23. B.C. Khoo, T.G. Liu and K.S. Yeo, Ghost fluid method for strong shock impacting on material interface, J. Comput. Phys. 190 (2003), 651-681.

24. R.J. LeVeque and Z.L. Li, The immersed interface method for elliptic equations with discontinuous coefficients and singular sources, SIAM J. Numer. Anal. 31 (1994), 1019-1044. MR 1286215 (95g:65139)

25. J. Li, J.M. Melenk, B. Wohlmuth and J. Zou, Optimal a priori estimates for higher order finite element methods for elliptic interface problems, Applied Numerical Mathematics (in press) (2009).

26. Z.L. Li, A fast iterative algorithm for elliptic interface problems, SIAM J. Numer. Anal. 35 (1998), 230-254. MR1618460 (99b:65126)

27. Z.L. Li and K. Ito, Maximum principle preserving schemes for interface problems with discontinuous coefficients, SIAM J. Sci. Comput. 23 (2001), 339-361. MR1860918 (2002h:65166)

28. Z.L. Li, T. Lin and X. Wu, New Cartesian grid methods for interface problems using the finite element formulation, Numer. Math. 96 (2003), 61-98. MR2018791(2005c:65104)

29. J.L. Lions and E. Magenes, Non-Homogeneous Boundary Value Problems and Applications, Springer-Verlag, Berlin, 1972.

30. X.D. Liu, R.P. Fedkiw and M.J. Kang, A boundary condition capturing method for Poisson's equation on irregular domains, J. Comput. Phys. 160 (2000), 151-178. MR 1756763 (2001a:65152)

31. X.D. Liu and T. C. Sideris, Convergence of the ghost fluid method for elliptic equations with interfaces, Math. Comput. 72 (2003), 1731-1746. MR1986802(2004h:65107)

32. H. Kang and J.K. Seo, The layer potential technique for the inverse conductivity problem, Inverse Problems 12 (1996) 267-278. MR1391539 (97d:35242)

33. H. Kang, J.K. Seo and D. Sheen, Numerical identification of discontinuous conductivity coefficients, Inverse Problems 13 (1997) 113-123. MR1435871(98k:78052) 
34. W. McLean, Strongly elliptic systems and boundary integral equations, Cambridge University Press, Cambridge, UK (2000). MR1742312 (2001a:35051)

35. H. Owhadi and L. Zhang, Metric based up-scaling, Comm. Pure Appl. Math 60 (2007) 675723. MR2292954(2008c:35041)

36. C. Pechstein and R. Scheichl, Analysis of FETI methods for multiscale PDEs, Numer. Math. 111 (2008), 293-333. MR2456834

37. C.S. Peskin, Numerical study of blood flow in the heart, J. Comput. Phys. 25 (1977), 220-252. MR 0490027 (58:9389)

38. C.S. Peskin, The immersed boundary method, Acta Numerica 11 (2002), 479-517. MR2009378 (2004h:74029)

39. M. Plum and C. Wieners, Optimal a priori estimates for interface problems, Numer. Math. 95 (2003), 735-759. MR2013126 (2004i:65126)

40. M.E. Taylor, Partial Differential Equations, Volume I, Springer-Verlag, Berlin, 1996. MR:1395147 (98b:35002a)

41. A. Toselli and O. Widlund, Domain Decomposition Methods Algorithms and Theory, SpringerVerlag, Berlin-Heidelberg-New York, 2005. MR 2104179 (2005g:65006)

42. S.O. Unverdi and G. Tryggvason, A front-tracking method for viscous, incompressible, multifluid flows, J. Comput. Phys. 100 (1992), 25-37.

43. T. von Petersdorff, Randwertprobleme der Elastizitätstheorie für Polyeder Singularitätaten und Approximation mit Randelementmethoden, Ph.D. Thesis, T.U. Darmstadt, German, 1989.

44. M. Vohralík, Guaranteed and fully robust a posteriori error estimates for conforming discretizations of diffusion problems with discontinuous coefficients, preprint, University of Paris VI, 2008. MR 2451464

45. Y.C. Zhou, S. Zhao, M. Feig and G.W. Wei, High order matched interface and boundary method for elliptic equations with discontinuous coefficients and singular sources, J. Comp. Phys. 213 (2006), 1-30. MR2186592(2007e:65137)

Department of Applied and Computational Mathematics, California Institute of Technology, Pasadena, California 91125

E-mail address: ccchu@acm.caltech.edu

Current address: Department of Mathematics, University of Texas at Austin, 1 University Station C1200, Austin, Texas 78712

Department of Mathematical Sciences, University of Bath, Bath BA2 7Ay, United KINGDOM

E-mail address: I.G.Graham@bath.ac.uk

Department of Applied and Computational Mathematics, California Insitute of Technology, Pasadena, California 91125

E-mail address: hou@acm.caltech.edu 